\title{
Irreducibility of inverse limits on intervals
}

\author{
by
}

David J. Ry den (Rolla, MO)

\begin{abstract}
A procedure for obtaining points of irreducibility for an inverse limit on intervals is developed. In connection with this, the following are included. A semiatriodic continuum is defined to be a continuum that contains no triod with interior. Characterizations of semiatriodic and unicoherent continua are given, as well as necessary and sufficient conditions for a subcontinuum of a semiatriodic and unicoherent continuum $M$ to lie within the interior of a proper subcontinuum of $M$.
\end{abstract}

1. Introduction. In 1944, R. H. Sorgenfrey [8] generalized a theorem of R. L. Moore by showing that every unicoherent continuum that is not a triod is irreducible. Sorgenfrey's result is one of existence only; it does not provide a means for obtaining two points between which such a continuum is irreducible. In 1951, R. H. Bing [2] defined snake-like continua (which are often called chainable continua) and noted that they are hereditarily unicoherent and atriodic. In 1959, J. R. Isbell [4] showed that every chainable continuum is homeomorphic to an inverse limit on intervals. That the converse is true is not difficult to prove. It follows from these results that every continuum expressed as an inverse limit on intervals is irreducible. In 1969, D. P. Kuykendall [5] gave in his master's thesis a fairly simple procedure for finding a pair of points between which an inverse limit on intervals with a single bonding map is irreducible. This procedure makes use of the fixed points and period-two

2000 Mathematics Subject Classification: Primary 54F15; Secondary 54F55, 54F65, 54F99.

Key words and phrases: continuum, irreducible, inverse limit, chainable, triod, unicoherent, indecomposable, absolutely terminal subcontinuum.

The material in this paper appears in the author's dissertation, directed by W. T. Ingram, in partial fulfillment of the requirements for the degree Doctor of Philosophy at the University of Missouri-Rolla; portions of this paper were presented at the Spring Topology and Dynamics Conference in Fairfax, VA, March 12-14, 1998. The author wishes to acknowledge support under a Chancellor's fellowship provided by the University of Missouri-Rolla. 
points of the bonding map; it does not extend to the general case, however. In 1973, Kuykendall [6] gave necessary and sufficient conditions for an inverse limit on metric continua to be irreducible about a given finite set of points. However, there was still no procedure for finding their coordinates.

The goal of this paper is to find, for a continuum expressed as an inverse limit on intervals, coordinates of two points between which the continuum is irreducible. The solution given includes results on other continuum theoretic problems that do not involve inverse limits. Variations on the following question are considered: Under what conditions does a proper subcontinuum of a chainable continuum fail to lie in the interior of any other proper subcontinuum? The answer given remains true if "chainable continuum" is replaced with "unicoherent continuum that contains no triod with nonvoid interior"; the solution presented is in this more general setting. Topological characterizations for unicoherent continua that contain no triod with nonvoid interior are also included, as well as various results about subcontinua that "lie at an end" of such a continuum.

A continuum is a compact connected subset of a metric space. A continuum is said to be irreducible between the two points $a$ and $b$ if and only if it contains both $a$ and $b$ but has no proper subcontinuum that contains both $a$ and $b$.

If $K$ is a subset of a continuum $M$, then $K$ is said to be a subset of $M$ with interior if it contains a nonempty open subset of $M$; otherwise, $K$ is said to be nowhere dense in $M$. A continuum $M$ is said to be a triod if and only if there is a subcontinuum $K$ of $M$ such that $M-K$ has at least three components. A continuum is said to be atriodic (semiatriodic) if and only if it contains no triod (triod with interior). A continuum $M$ is said to be unicoherent if and only if it is true that if $H$ and $K$ are subcontinua of $M$ such that $H \cup K=M$, then $H \cap K$ is connected. A continuum is said to be hereditarily unicoherent if and only if each of its subcontinua is unicoherent.

A continuum is said to be indecomposable if and only if it is not the union of two of its proper subcontinua; otherwise, it is said to be decomposable. A continuum $M$ is said to be two-indecomposable if and only if there are two indecomposable proper subcontinua of $M$ whose union is $M$.

A subcontinuum $K$ of a continuum $M$ is said to be an absolutely terminal subcontinuum of $M$ provided that for each pair of subcontinua of $M$ that contain $K$, one is a subset of the other. This definition is equivalent to the standard definition of "absolutely terminal continuum" given by D. E. Bennett and J. B. Fugate [1] save that it does not forbid a continuum to be an absolutely terminal subcontinuum of itself.

If $X_{1}, X_{2}, \ldots$ is a sequence of metric spaces, and for each positive integer $n, f_{n}$ is a continuous function from $X_{n+1}$ into $X_{n}$, the sequence $\left\{X_{n}, f_{n}\right\}$ is called an inverse sequence, the spaces $X_{n}$ are called factor spaces, and the 
functions $f_{n}$ are called bonding maps. The notation $f_{n}^{m}(m \geq n)$ denotes the identity on $X_{n}$ if $m=n, f_{n}$ if $m=n+1$, and the composition $f_{n}^{n+1} \circ f_{n+1}^{n+2} \circ$ $\ldots \circ f_{m-1}^{m}$ if $m$ is larger than $n+1$. The projection of the product space $\prod X_{n}$ into $X_{n}$, denoted by $\pi_{n}$, is the function from $\prod X_{n}$ into $X_{n}$ that satisfies $\pi_{n}(x)=x_{n}$ for each $x$ in $\prod X_{n}$. The inverse limit of the inverse sequence $\left\{X_{n}, f_{n}\right\}$, denoted by $\lim _{\longleftarrow}\left\{X_{n}, f_{n}\right\}$, is the subset of the product space $\prod X_{n}$ to which $x$ belongs if and only if $f_{n}\left(x_{n+1}\right)=x_{n}$ for each positive integer $n$. A continuum is chainable if and only if it is homeomorphic to the inverse limit of an inverse sequence for which each of the factor spaces is an interval. It is well known that chainable continua are atriodic and hereditarily unicoherent.

2. Points of irreducibility — part I. Every nondegenerate inverse limit on intervals can be rewritten as an inverse limit on intervals with surjective bonding maps; hence, surjectivity of the bonding maps will be assumed. In this section, candidates for points of irreducibility are given that, in general, will not suffice. Nevertheless, the unique subcontinuum of $M$ that is irreducible between them has some important properties that will be useful for obtaining points of irreducibility.

Notation. For any two points $a$ and $b$ of a hereditarily unicoherent continuum $M, \overline{a b}$ denotes the unique subcontinuum of $M$ that is irreducible between $a$ and $b$.

THEOREM 1. Suppose $\left\{\left[a_{n}, b_{n}\right], f_{n}\right\}$ is an inverse sequence with surjective bonding maps and $M$ is the inverse limit of $\left\{\left[a_{n}, b_{n}\right], f_{n}\right\}$. If $\left(\zeta_{1}, \omega_{1}\right)$, $\left(\zeta_{2}, \omega_{2}\right), \ldots$ is a sequence of points in $M \times M$ with the property that both $\pi_{n}\left(\zeta_{n}\right)=a_{n}$ and $\pi_{n}\left(\omega_{n}\right)=b_{n}$ for each positive integer $n$ and $(z, w)$ is a subsequential limit of $\left(\zeta_{1}, \omega_{1}\right),\left(\zeta_{2}, \omega_{2}\right), \ldots$, then no proper subcontinuum of $M$ contains $\overline{z w}$ in its interior.

Proof. Let $Y$ be a subcontinuum of $M$ that contains $\overline{z w}$ in its interior. Since $\operatorname{int}(Y) \times \operatorname{int}(Y)$ is an open set in $M \times M$ that contains the point $(z, w)$, $Y$ must contain both $z_{n}$ and $w_{n}$ for infinitely many positive integers $n$.

Therefore, $\pi_{n}(Y)=\left[a_{n}, b_{n}\right]$ for infinitely many positive integers $n$. It follows that $Y=M$.

It is worthwhile to note that in Theorem 1 , the continuum $\overline{z w}$ could be a proper or even degenerate subcontinuum of $M$. Consider the following example.

ExAmPLe. Let $f$ be the map of $[0,1]$ that satisfies $f(0)=1 / 2, f(1 / 4)=$ $0, f(3 / 4)=1$, and $f(1)=1 / 2$, and is linear on the intervals [0,1/4], $[1 / 4,3 / 4]$, and $[3 / 4,1]$. Let $M$ be the inverse limit of $\{[0,1], f\}$. Since $f(0)=f(1)=1 / 2$ and $f(1 / 2)=1 / 2$, it follows that $z=w=(1 / 2,1 / 2, \ldots)$. 
Hence the conclusion of Theorem 1, that no proper subcontinuum of $M$ contains $\overline{z w}$ in its interior, is insufficient to conclude that $M$ is irreducible between the points $z$ and $w$. Section 3 investigates the implications of a subcontinuum of $M$ failing to lie in the interior of any proper subcontinuum of $M$. In the context of the points $z$ and $w$, the results of Section 3 guarantee that every point of $M-\overline{z w}$ belongs to an indecomposable continuum "at the end" of $M$; in some sense, this reduces the problem of finding points of irreducibility for $M$ to that of finding points of irreducibility for an indecomposable inverse limit of intervals. Corollary 14.1 of Section 4 gives an algorithm for finding points of irreducibility in the indecomposable case, and the remainder of Section 4 is devoted to amalgamating this algorithm with that of Theorem 1 to produce a procedure for the general case. A summary of the general procedure appears after Theorem 19.

3. Absolutely terminal subcontinua in semiatriodic and unicoherent continua. The theorem from the last section raises the following question: under what conditions does a proper subcontinuum of a chainable continuum $M$ fail to lie in the interior of another proper subcontinuum of $M$ ? If $M$ is an arc, every proper subcontinuum of $M$ is contained in the interior of another proper subcontinuum of $M$. If $M$ is indecomposable, each of its proper subcontinua has void interior; hence, every proper subcontinuum of $M$ fails to lie in the interior of another proper subcontinuum of $M$. Indecomposability is not necessary, however. In Figure 1, the continuum

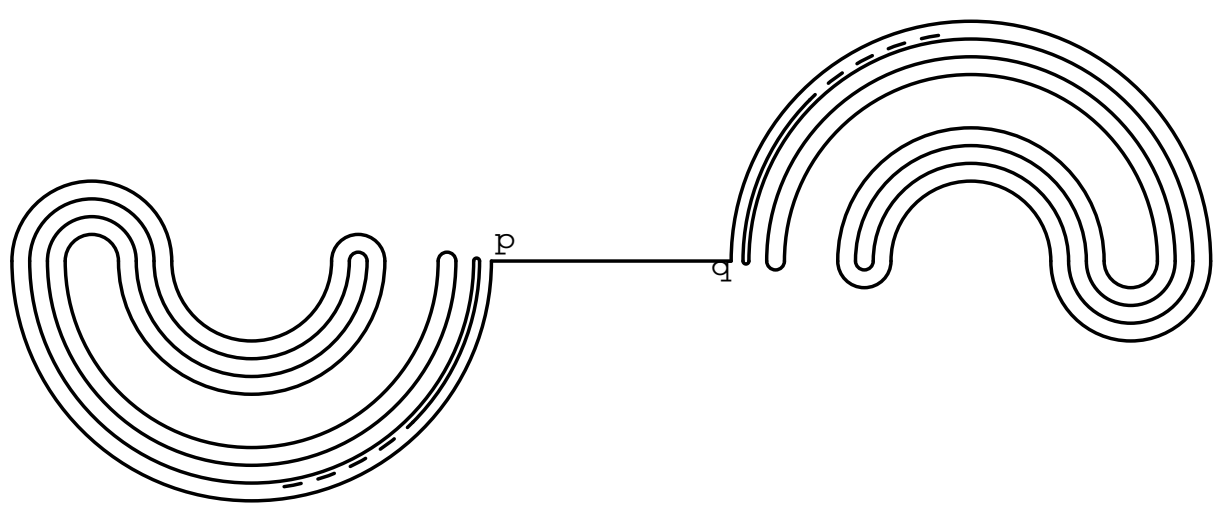

Fig. 1. Two BJK continua whose endpoints are joined by an arc

$M$ is two Brouwer-Janiszewski-Knaster continua whose endpoints, $p$ and $q$, are joined by an arc. No proper subcontinuum of $M$ contains this arc in its interior. These examples suggest that the answer to the above question may be related to the presence of indecomposable subcontinua of $M$ that "lie at an end" of $M$ or to the indecomposability of $M$ itself. Section 3.3 confirms this 
for $M$ belonging to the larger class of semiatriodic and unicoherent continua. It is first necessary to consider some properties of continua belonging to this class and of their absolutely terminal subcontinua (defined below).

3.1. Semiatriodic and unicoherent continua. The following two results may be found with proofs in [3, Theorem 3.1] and [7, Theorem 2.7] respectively. The theorem of W. Dwayne Collins shows that the class of semiatriodic and unicoherent continua is the same as the class of continua for which every subcontinuum with interior is unicoherent and fails to be a triod.

ThEOREM 2 (Collins, [3]). If $M$ is a semiatriodic continuum, then every proper subcontinuum of $M$ with interior is unicoherent.

TheOrem 3 (Owens, [7]). Suppose $M$ is a continuum, and suppose $A$ and $B$ are subcontinua of $M$ with a common point. If $A$ is an absolutely terminal subcontinuum of $M$, then $A \cap B$ is connected.

TheOREM 4. Suppose $M$ is a continuum. Each of the following statements is equivalent to the condition that $M$ is semiatriodic and unicoherent.

(1) If $A$ is a subcontinuum of $M$, and $C$ is a component of $M-A$, then $\bar{C}$ is an absolutely terminal subcontinuum of $M$.

(2) If $A$ is a proper subcontinuum of $M$, and $B$ is a subcontinuum of $M$ such that $A \cup B=M$, then $B$ is an absolutely terminal subcontinuum of $M$.

Proof. First, it is shown that $M$ is semiatriodic and unicoherent if and only if (1) holds. Suppose that $M$ is semiatriodic and unicoherent, but that there is a subcontinuum $A$ of $M$ and a component $C$ of $M-A$ such that $\bar{C}$ is not an absolutely terminal subcontinuum of $M$. Then there exist two subcontinua, $C_{1}$ and $C_{2}$, of $M$, each containing $\bar{C}$, such that $C_{1}$ does not contain $C_{2}$ and $C_{2}$ does not contain $C_{1}$. It will be shown that $C_{1} \cup C_{2}$ is a triod. Denote $M-C$ by $C^{\mathrm{c}}$, and let $D=C^{\mathrm{c}} \cap C_{1} \cap C_{2}$. Note that $C$ misses $A$ and that $M$ is not a triod; hence, $C$ is a proper open subset of $M$. Since $M$ is connected, it follows that $\bar{C}-C$ is nonempty; furthermore, $\bar{C}-C \subset C^{\mathrm{c}} \cap C_{1} \cap C_{2}$, so $D$ is also nonempty. Since $M$ is not a triod, either $C^{\mathrm{c}}$ is $A$, or $C^{\mathrm{c}}$ is the union of $A$ with a single component of $M-A$; hence, $C^{\mathrm{c}}$ is a continuum. Note that $C^{\mathrm{c}} \cup C_{1}=M$. Since $M$ is unicoherent, $C^{\mathrm{c}} \cap C_{1}$ is a continuum. Then $\left(C^{\mathrm{c}} \cap C_{1}\right) \cup C_{2}$ is a subcontinuum of $M$ that contains the open set $C$. It follows from Theorem 2 that $D$ is a continuum. Consider the sets $C_{1} \cap C^{\mathrm{c}}-D, C_{2} \cap C^{\mathrm{c}}-D$, and $C_{1} \cap C_{2}-D$. Since $C \subset C_{1} \cap C_{2}$, it follows that $D \cup\left(C_{1} \cap C^{\mathrm{c}}-D\right) \cup\left(C_{2} \cap C^{\mathrm{c}}-D\right) \cup\left(C_{1} \cap C_{2}-D\right)=$ $\left[\left(C_{1} \cup C_{2}\right) \cap C^{\mathrm{c}}\right] \cup\left(C_{1} \cap C_{2}\right)=C_{1} \cup C_{2}$. The closed set, $C_{2}$, does not intersect $C_{1} \cap C^{\mathrm{c}}-D$; hence, $C_{1} \cap C^{\mathrm{c}}-D$ contains no point or limit point of $\left(C_{2} \cap C^{\mathrm{c}}-D\right) \cup\left(C_{1} \cap C_{2}-D\right)$. Similarly, $C_{2} \cap C^{\mathrm{c}}-D$ contains no point or limit point of $\left(C_{1} \cap C^{\mathrm{c}}-D\right) \cup\left(C_{1} \cap C_{2}-D\right)$, and $C_{1} \cap C_{2}-D$ contains no 
point or limit point of $\left(C_{1} \cap C^{\mathrm{c}}-D\right) \cup\left(C_{2} \cap C^{\mathrm{c}}-D\right)$. Therefore, $C_{1} \cup C_{2}$ is a triod; furthermore, $C_{1} \cup C_{2}$ contains the open set $C$. This is a contradiction with the assumption that $M$ is semiatriodic.

Suppose that $M$ is not unicoherent. Then there exist proper subcontinua, $M_{1}$ and $M_{2}$, of $M$ such that $M_{1} \cup M_{2}=M$, and $M_{1} \cap M_{2}$ is not connected. Let $C$ be a component of $M-M_{1}$. Then $\bar{C}$ is a subcontinuum of $M_{2}$. By Theorem $3, M_{2}$ is not an absolutely terminal continuum of $M$; therefore, $\bar{C}$ is not an absolutely terminal subcontinuum of $M$, and (1) does not hold.

Suppose that $M$ contains a triod, $T$, with interior. There is a subcontinuum, $D$, of $T$ such that $T-D$ is the union of three mutually separated sets, $T_{1}, T_{2}$, and $T_{3}$.

CASE (i). Suppose $D$ has interior relative to $M$. If $M-D$ is connected, and $C$ is a component of $M-\overline{M-D}$, then $T_{1} \cup D$ and $T_{2} \cup D$ are both continua that contain $\bar{C}$, but neither is a subset of the other. Thus (1) does not hold. If $M-D$ is not connected, then it is the union of two mutually separated sets, $H$ and $K$. One of $H$ and $K$ contains two components of $T-D$. Renaming if necessary, assume $H$ contains two components of $T-D$. Note that $H \cup D$ and $K \cup D$ are proper subcontinua of $M$. Let $C$ be a component of $M-(H \cup D)$. Then $K \cup D$ is a subcontinuum of $M$ that contains $\bar{C}$. Since $H$ contains two components of $T-D, K \cup D$ is not an absolutely terminal subcontinuum of $M$. Since $K \cup D$ contains $\bar{C}, \bar{C}$ is not an absolutely terminal subcontinuum of $M$, and (1) does not hold.

CAse (ii). Suppose $D$ does not have interior relative to $M$. Then one of $T_{1}, T_{2}$, and $T_{3}$, say $T_{1}$, has interior relative to $M$. If $M-T_{1}$ is connected, and $C$ is a component of $M-\overline{M-T_{1}}$, then $T_{1} \cup D \cup T_{2}$ and $T_{1} \cup D \cup T_{3}$ are both continua that contain $\bar{C}$, but neither is a subset of the other, so (1) does not hold. If $M-T_{1}$ is not connected, then it is the union of two mutually separated sets, $H$ and $K$. Since $T-T_{1}$ is connected, it is a subset of either $H$ or $K$. Renaming if necessary, assume $H$ contains $T-T_{1}$. Note that $H \cup T_{1}$ and $K \cup T_{1}$ are proper subcontinua of $M$. Let $C$ be a component of $M-\left(H \cup T_{1}\right)$. Then $K \cup T_{1}$ is a subcontinuum of $M$ that contains $\bar{C}$. Each of $D, T_{2}$, and $T_{3}$ is contained by $H$, so $\left(K \cup T_{1}\right) \cup D \cup T_{2}$ and $\left(K \cup T_{1}\right) \cup D \cup T_{3}$ are subcontinua of $M$ that contain $\bar{C}$, but neither is a subset of the other. Thus, (1) does not hold.

To show that (1) implies (2), suppose $A$ is a proper subcontinuum of $M$ and $B$ is a subcontinuum of $M$ such that $A \cup B=M$. Let $C$ be a component of $M-A$. Then $\bar{C}$ is a subcontinuum of $B$. By (1), $\bar{C}$ is an absolutely terminal subcontinuum of $M$; hence, $B$ is an absolutely terminal subcontinuum of $M$.

Finally, suppose (2) holds. Let $A$ be a subcontinuum of $M$. Suppose $M-A$ is the union of three mutually separated sets, $H, J$, and $K$. Then 
$A \cup H \cup J$ is a proper subcontinuum of $M$, and $A \cup K$ is a subcontinuum of $M$ such that $(A \cup H \cup J) \cup(A \cup K)=M$. Hence, by (2), $A \cup K$ is an absolutely terminal subcontinuum of $M$; but $(A \cup K) \cup H$ and $(A \cup K) \cup J$ are subcontinua of $M$ neither of which contains the other. Therefore, $M-A$ has at most two components. It follows that each component of $M-A$ is open. Let $C$ be a component of $M-A$. Since $C$ is open, $C^{\mathrm{c}}$ is a proper subcontinuum of $M$. Then, by (2), $\bar{C}$ is an absolutely terminal subcontinuum of $M$. Therefore, (2) implies (1).

\subsection{Properties of absolutely terminal subcontinua}

TheOREM 5. Suppose $M$ is a semiatriodic and unicoherent continuum, and suppose $A$ is a proper subcontinuum of $M$ with interior. The following are equivalent:

(1) $A$ is an absolutely terminal subcontinuum of $M$.

(2) $M-A$ is connected.

(3) $\overline{\operatorname{int}(A)}$ is an absolutely terminal subcontinuum of $M$.

Proof. First, suppose (2) does not hold. Then $M-A$ has two components, $H$ and $K$. Each of $A \cup H$ and $A \cup K$ is a subcontinuum of $M$ that contains $A$; but neither of them is a subset of the other. Therefore, (1) does not hold.

Next, it is shown that (2) implies (3). By (1) of Theorem $4, \overline{M-A}$ is an absolutely terminal subcontinuum of $M$ with nonempty interior. It has been shown that (1) implies (2), so $M-\overline{M-A}$ is connected. Therefore, $\overline{\operatorname{int}(A)}=\overline{M-\overline{M-A}}$ is an absolutely terminal subcontinuum of $M$ by (1) of Theorem 4.

Finally, $A$ is a continuum that contains $\overline{\operatorname{int}(A)}$, so (3) implies (1).

TheOREM 6. Suppose $M$ is a semiatriodic and unicoherent continuum. If $A$ and $B$ are absolutely terminal subcontinua of $M$ with interior that have a common point, then either $A \cup B=M$ or one of $A$ and $B$ is a subset of the other.

Proof. Suppose $A \cup B$ is not all of $M$, and denote $M-(A \cup B)$ by $C$. Since $A \cup B$ is an absolutely terminal subcontinuum of $M$ with interior, $C$ is connected by Theorem 5 . Then $\bar{C}$ is an absolutely terminal subcontinuum with interior by (1) of Theorem 4 .

Case (i). Suppose both $A$ and $B$ intersect $\bar{C}$. Then each of $\bar{C} \cup A$ and $\bar{C} \cup B$ is a continuum containing $\bar{C}$, so one of them is a subset of the other. Renaming if necessary, assume $\bar{C} \cup A \subset \bar{C} \cup B$. It follows that $\operatorname{int}(A \cup B) \cap$ $(\bar{C} \cup A) \subset \operatorname{int}(A \cup B) \cap(\bar{C} \cup B)$; but $\operatorname{int}(A \cup B) \cap \bar{C}$ is empty, so $\overline{\operatorname{int}(A)} \subset$ $\overline{\operatorname{int}(A \cup B) \cap A} \subset \overline{\operatorname{int}(A \cup B) \cap B} \subset B$. Each of $A$ and $B$ is a continuum 
that contains $\overline{\operatorname{int}(A)}$, and $\overline{\operatorname{int}(A)}$ is an absolutely terminal subcontinuum by Theorem 5; therefore, one of $A$ and $B$ is a subset of the other.

Case (ii). Suppose only one of $A$ and $B$ intersects $\bar{C}$. Renaming if necessary, assume $A$ intersects $\bar{C}$. Then each of $A \cup B$ and $A \cup \bar{C}$ is a continuum containing $A$, so one of them is a subset of the other. The continuum $\bar{C}$ is not a subset of $A \cup B$, so $A \cup B \subset A \cup \bar{C}$. Denote $M-A$ by $A^{\mathrm{c}}$. Then $A^{\mathrm{c}} \cap B \subset A^{\mathrm{c}} \cap \bar{C}$, but $B$ does not intersect $\bar{C}$, so $A^{\mathrm{c}} \cap B$ is empty. It follows that $B \subset A$.

TheOREM 7. Suppose $M$ is a semiatriodic and unicoherent continuum. If each of $A, B$, and $C$ is an absolutely terminal subcontinuum of $M$ with interior, then one of $A, B$, and $C$ is a subset of some other one of $A, B$, and $C$.

Proof. The result is trivial if one of $A, B$, and $C$ is all of $M$; hence, it is assumed that $A, B$, and $C$ are proper subcontinua of $M$. Then $M-A, M-B$, and $M-C$ are connected by Theorem 5 , so $\overline{M-A}, \overline{M-B}$, and $\overline{M-C}$ are absolutely terminal subcontinua of $M$ with interior by (1) of Theorem 4 . Suppose $A, B$, and $C$ are pairwise disjoint. Since $M$ is connected, $A \cup B \cup C$ is not all of $M$. Each of $\overline{M-A}, \overline{M-B}$, and $\overline{M-C}$ contains the open set $M-(A \cup B \cup C)$. Hence, $\overline{M-A} \cup \overline{M-B}$ is a proper subcontinuum of $M$ with interior. Therefore, by Theorem $2, \overline{M-A} \cap \overline{M-B}$ is a subcontinuum of $M$. It follows further that $(\overline{M-A} \cap \overline{M-B}) \cup \overline{M-C}$ is a subcontinuum of $M$ with interior, and, hence, that $D=(\overline{M-A}) \cap(\overline{M-B}) \cap(\overline{M-C})$ is a subcontinuum of $M$ with interior. Note that $M-D=(M-\overline{M-A}) \cup(M-\overline{M-B}) \cup$ $(M-\overline{M-C})=\operatorname{int}(A) \cup \operatorname{int}(B) \cup \operatorname{int}(C)$. This is a contradiction since $A, B$, and $C$ are assumed to be pairwise disjoint closed sets and $M$ is not a triod.

Renaming if necessary, assume $A$ intersects $B$. If one of $A$ and $B$ is a subset of the other, the conclusion of the theorem follows; otherwise, $A \cup B=$ $M$ by Theorem 6 . Then one of $A$ and $B$ intersects $C$. Again renaming if necessary, assume $C$ intersects $A$. If one of $A$ and $C$ is a subset of the other, the conclusion of the theorem follows; otherwise, $A \cup C=M$. Then $B$ and $C$ are both continua that contain the absolutely terminal subcontinuum $\overline{M-A}$; hence, one of $B$ and $C$ is a subset of the other.

COROLlary 7.1. If $M$ is a semiatriodic and unicoherent continuum, then $M$ has at most two indecomposable absolutely terminal subcontinua with interior.

Proof. Suppose $M$ has three indecomposable absolutely terminal subcontinua with interior. Then, by Theorem 7, one of them has a proper subcontinuum with interior, which is not possible.

EXAMPLE. Let $T_{1}$ denote the set of points $(x, y)$ in the plane such that $x \in[-1,0]$ and $y=0, T_{2}$ denote the set of points $(x, y)$ such that $x=0$ and 
$y \in[-1,1]$, and $T_{3}$ denote the set of points $(x, y)$ such that $x \in(0,1]$ and $y=\sin (1 / x)$. Consider the continuum $T_{1} \cup T_{2} \cup T_{3}$, and note that $T_{1} \cup T_{2}$ is a triod that has interior in $T_{1} \cup T_{2} \cup T_{3}$. The statements of Theorems 5 , 6 , and 7 do not remain true if "semiatriodic and unicoherent continuum" is replaced by "unicoherent continuum that is not a triod". Counterexamples may be obtained by considering the continuum $T_{1} \cup T_{2} \cup T_{3}$.

\subsection{Proper subcontinua and their interior}

Lemma 1. Suppose $M$ is a continuum that is not a triod, and suppose $B$ is an absolutely terminal subcontinuum of $M$. If there is a subset, $A$, of $M-B$ such that $\bar{A}$ is a decomposable subcontinuum of $M$ with interior, then there is a proper subcontinuum of $M$ that contains $B$ in its interior.

Proof. Let $A_{1}$ and $A_{2}$ be proper subcontinua of $\bar{A}$ whose union is $\bar{A}$. Since $\bar{A}$ has interior, one of $A_{1}$ and $A_{2}$ has interior. Renaming if necessary, assume $A_{1}$ has interior. Each component of $M-A_{1}$ is open since $M$ is not a triod. Therefore, if $A_{1}$ fails to intersect $B$, the closure of the component of $M-A_{1}$ that contains $B$ is a proper subcontinuum of $M$ that contains $B$ in its interior. Suppose $A_{1}$ and $B$ have a common point. Then $A_{1} \cup B$ is a continuum. Since $A_{1}$ is a proper subcontinuum of $\bar{A}, A_{1}$ cannot contain every point of $A$. It follows that $A_{1} \cup B$ is a proper subcontinuum of $M$. Note that $A_{1} \cup B$ is an absolutely terminal subcontinuum of $M$, since it contains $B$. Therefore, $M-\left(A_{1} \cup B\right)$ is connected. Since $B$ is an absolutely terminal subcontinuum of $M$, and $A_{1}$ intersects $B, \overline{M-\left(A_{1} \cup B\right)}$ cannot intersect $B$. It follows that $A_{1} \cup B$ contains $B$ in its interior.

TheOREM 8. Suppose $M$ is a semiatriodic and unicoherent continuum. The following are equivalent:

(1) $M$ contains a nowhere dense subcontinuum that is not contained in the interior of any proper subcontinuum of $M$.

(2) $M$ is either indecomposable or two-indecomposable.

Proof. Suppose $M$ is neither indecomposable nor two-indecomposable, and let $X$ be a nowhere dense subcontinuum of $M$. Let $M_{0}$ be a proper subcontinuum of $M$ with interior, and let $M_{1}$ be the closure of some component of $M-M_{0}$. By (1) of Theorem $4, M_{1}$ is an absolutely terminal subcontinuum of $M$ with interior. By Theorem 5, $M-M_{1}$ is connected. Let $M_{2}=\overline{M-M_{1}}$. It follows from (1) of Theorem 4 that $M_{2}$ is an absolutely terminal subcontinuum of $M$; furthermore, $M_{2}$ has interior. Since $\overline{M-\left(M_{1} \cap M_{2}\right)}=\overline{M-M_{1}} \cup \overline{M-M_{2}}=M_{2} \cup \overline{M-M_{2}}=M$, it follows that $M_{1} \cap M_{2}$ is nowhere dense. One of $M_{1}$ and $M_{2}$ is decomposable since $M_{1} \cup M_{2}=M$. It is assumed that $M_{1}$ is decomposable, the remainder of the proof is the same if $M_{2}$ is decomposable. 
Let $B=M_{2} \cup X$, and let $A=M_{1}-\left(M_{1} \cap B\right)$. Since each of $M_{1} \cap M_{2}$ and $M_{1} \cap X$ is nowhere dense, their union, $M_{1} \cap B$, is nowhere dense. Therefore, $\overline{\operatorname{int}\left(M_{1}\right)-\left(\operatorname{int}\left(M_{1}\right) \cap\left(M_{1} \cap B\right)\right)}=\overline{\operatorname{int}\left(M_{1}\right)}=M_{1}$, and so

$$
\begin{aligned}
M_{1} & =\overline{\operatorname{int}\left(M_{1}\right)-\left(\operatorname{int}\left(M_{1}\right) \cap\left(M_{1} \cap B\right)\right)} \\
& =\overline{\operatorname{int}\left(M_{1}\right) \cap\left(\left(M_{1}\right)-\left(M_{1} \cap B\right)\right)}=\overline{M_{1}-\left(M_{1} \cap B\right)} \subset M_{1} .
\end{aligned}
$$

It follows that $\bar{A}=M_{1}$ and, hence, that $\bar{A}$ is a decomposable subcontinuum of $M$ with interior. If $B$ is not connected, then $X$ misses $M_{2}$ and, hence, $M_{1}$ is a proper subcontinuum of $M$ that contains $X$ in its interior. If $B$ is connected, then $B$ is an absolutely terminal subcontinuum of $M$ since it contains the absolutely terminal subcontinuum $M_{2}$; furthermore, $A \subset M-B$. Therefore, by Lemma 1 , there is a proper subcontinuum of $M$ that contains $B$ and, hence, $X$ in its interior.

If $M$ is indecomposable, then every proper subcontinuum of $M$ is a nowhere dense subcontinuum of $M$ that is not contained in the interior of any proper subcontinuum of $M$. Suppose $M$ is two-indecomposable. Let $M_{1}$ and $M_{2}$ be proper indecomposable subcontinua of $M$ whose union is $M$. The continuum $M$ is unicoherent, so $M_{1} \cap M_{2}$ is a continuum; furthermore, $M_{1} \cap M_{2}$ is a proper subcontinuum of the indecomposable continuum $M_{1}$, so $M_{1} \cap M_{2}$ is nowhere dense. It is shown that there is no proper subcontinuum of $M$ that contains $M_{1} \cap M_{2}$ in its interior. Assume the contrary, and let $Y$ be a proper subcontinuum of $M$ that contains $M_{1} \cap M_{2}$ in its interior. Let $A$ be a component of $M-Y$. The sets $M_{1}-\left(M_{1} \cap M_{2}\right)$ and $M_{2}-\left(M_{1} \cap M_{2}\right)$ are mutually separated, so $A$ is a subset of one of them. Renaming if necessary, assume $A \subset M_{1}-\left(M_{1} \cap M_{2}\right)$. Then $A \subset M_{1}$; furthermore, $\bar{A}$ is not all of $M_{1}$ since $\bar{A} \subset M_{1}-\left(M_{1} \cap \operatorname{int}(Y)\right) \subset M_{1}-\left(M_{1} \cap M_{2}\right)$. Hence, $\bar{A}$ is a proper subcontinuum of $M_{1}$ with interior. This is a contradiction since $M_{1}$ is indecomposable. Therefore, no proper subcontinuum of $M$ contains $M_{1} \cap M_{2}$ in its interior.

TheOREM 9. Suppose $M$ is a semiatriodic and unicoherent continuum, and suppose $X$ is a proper subcontinuum of $M$ with interior. The following are equivalent:

(1) No proper subcontinuum of $M$ contains $X$ in its interior.

(2) The closure of each component of $M-X$ is indecomposable.

(3) Every point of $M-X$ belongs to an indecomposable absolutely terminal subcontinuum of $M$ that has interior and contains a point of $X$.

(4) Every subcontinuum of $M$ with interior contains a point of $X$.

Proof. It is first shown that (1) implies (2). Suppose (2) does not hold. Then there is a component, $A$, of $M-X$ with the property that $\bar{A}$ is decomposable. If $M-X$ has another component, let $B$ be the union of $X$ with this component; otherwise, let $B=X$. Then $B$ is an absolutely 
terminal subcontinuum by (1) of Theorem 4 or by Theorem 5 respectively. Since $A$ is a subset of $M-B$, and $\bar{A}$ is a decomposable subcontinuum of $M$ with interior, it follows from Lemma 1 that there is a proper subcontinuum of $M$ that contains $B$ and, hence, $X$ in its interior.

That (2) implies (3) follows from (1) of Theorem 4.

To see that (3) implies (4), suppose (4) does not hold. Then $M$ has a subcontinuum, $Y$, with nonempty interior that does not intersect $X$. If $M-Y$ has two components, let $Y_{1}$ be the union of $Y$ with the component of $M-Y$ that does not intersect $X$; otherwise, let $Y_{1}=Y$. In either case, $Y_{1}$ is an absolutely terminal subcontinuum by (1) of Theorem 4 or by Theorem 5 respectively, $Y_{1}$ has interior, and $Y_{1}$ does not intersect $X$. If $M-X$ has two components, let $X_{1}$ be the union of $X$ with the component of $M-X$ that does not intersect $Y_{1}$; otherwise, let $X_{1}=X$. Then $X_{1}$ is an absolutely terminal subcontinuum by (1) of Theorem 4 or by Theorem 5 respectively, $X_{1}$ has nonempty interior, $X_{1}$ contains $X$, and $X_{1}$ does not intersect $Y_{1}$. Since $M$ is connected, $X_{1} \cup Y_{1}$ cannot be all of $M$. Let $z$ be a point of $M-\left(X_{1} \cup Y_{1}\right)$. Suppose there is an indecomposable absolutely terminal subcontinuum, $Z$, of $M$ with interior that contains $z$. Then by Theorem 7 , one of $X_{1}, Y_{1}$, and $Z$ is a subset of some other one of $X_{1}, Y_{1}$, and $Z$. Since $z$ is in neither $X_{1}$ nor $Y_{1}, Z$ cannot be a subset of either $X_{1}$ or $Y_{1}$; furthermore, $X_{1}$ and $Y_{1}$ are mutually exclusive. Therefore, one of $X_{1}$ and $Y_{1}$ is a proper subset of $Z$. This is a contradiction since both $X_{1}$ and $Y_{1}$ have interior, and $Z$ is assumed to be indecomposable. Therefore, there is no indecomposable absolutely terminal subcontinuum of $M$ with interior that contains $z$. Since $z$ is not in $X$, it follows that (3) does not hold.

Finally, it is shown that (4) implies (1). Suppose there is a proper subcontinuum, $Y$, of $M$ that contains $X$ in its interior. Let $A$ be a component of $M-Y$. Then $\bar{A}$ is a subcontinuum of $M$ with interior that fails to intersect $X$.

THEOREM 10. Suppose $M$ is a semiatriodic and unicoherent continuum, and suppose $X$ is a proper subcontinuum of $M$ with interior. The following are equivalent:

(1) There is a proper subcontinuum of $M$ that contains $X$ in its interior.

(2) The closure of some component of $M-X$ is decomposable.

(3) There is a subset, $A$, of $M-X$ such that $\bar{A}$ is a decomposable subcontinuum of $M$ with interior.

Proof. It follows from Theorem 9 that (1) implies (2), and (3) follows directly from (2). Suppose (3) holds. It is shown that $A$ cannot intersect two components of $M-X$. Suppose the contrary. Let $Q$ be the closure of one of these components. Then $Q$ is an absolutely terminal subcontinuum of $M$ by (1) of Theorem 4. Since $Q \cup X$ and $Q \cup \bar{A}$ are both continua that contain the 
absolutely terminal subcontinuum $Q$, one of $Q \cup X$ and $Q \cup \bar{A}$ is a subset of the other. By assumption, $A$ intersects a component of $M-X$ different from $Q$, so $A$ intersects $M-(Q \cup X)$. It follows that $(Q \cup X) \subset(Q \cup \bar{A})$. Then $\operatorname{int}(X) \cap(Q \cup X) \subset \operatorname{int}(X) \cap(Q \cup \bar{A})$. Since $Q \cap \operatorname{int}(X)$ is empty, $\operatorname{int}(X) \subset \bar{A}$. This is not possible since $A \subset M-X$. Therefore, $A$ cannot intersect two components of $M-X$. If $M-X$ has two components, let $B$ be the union of $X$ with the one that does not intersect $A$; otherwise, let $B=X$. Then $B$ is an absolutely terminal subcontinuum of $M$ by (1) of Theorem 4 or by Theorem 5 respectively, and $A$ is a subset of $M-B$. Therefore, by Lemma 1 there is a proper subcontinuum of $M$ that contains $B$ and, hence, $X$ in its interior.

THEOREM 11. Suppose $M$ is a semiatriodic and unicoherent continuum. The following are equivalent:

(1) Every proper subcontinuum of $M$ is contained in the interior of some proper subcontinuum of $M$.

(2) Every absolutely terminal subcontinuum of $M$ with interior is decomposable.

Pr oof. Suppose there is an absolutely terminal subcontinuum, $Q$, with interior that is indecomposable. If $Q=M$, then no proper subcontinuum of $M$ has interior, and, hence, (1) does not hold. Suppose $Q$ is a proper subcontinuum of $M$. Then $M-Q$ is connected by Theorem 5 , and $\overline{M-Q}$ is an absolutely terminal subcontinuum of $M$ with interior by (1) of Theorem 4. By Theorem 5 again, $M-\overline{M-Q}$ is connected. Note that $\overline{M-\overline{M-Q}}=\overline{\operatorname{int}(Q)} \subset Q$. No proper subcontinuum of $Q$ has nonempty interior, so $\overline{M-\overline{M-Q}}=Q$. Therefore, the only component of $M-\overline{M-Q}$ has an indecomposable closure. Hence, by Theorem 9 , no proper subcontinuum of $M$ contains $\overline{M-Q}$ in its interior.

Suppose every absolutely terminal subcontinuum of $M$ with interior is decomposable. Let $X$ be a proper subcontinuum of $M$.

CASE (i). Suppose $X$ has interior. Let $A$ be a component of $M-X$. Then $\bar{A}$ is an absolutely terminal subcontinuum of $M$ by (1) of Theorem 4 , and $A$ has interior since $M$ is not a triod, so $\bar{A}$ is decomposable. Then, by Theorem 9 , there is a proper subcontinuum of $M$ that contains $X$ in its interior.

CASE (ii). Suppose $X$ is nowhere dense. Since $M$ is an absolutely terminal subcontinuum of $M, M$ is decomposable. By (2) of Theorem $4, M$ cannot be two-indecomposable. Then, by Theorem 8 , there is a proper subcontinuum of $M$ that contains $X$ in its interior.

Corollary 11.1. Suppose $\left\{\left[a_{n}, b_{n}\right], f_{n}\right\}$ is an inverse sequence, $M$ is the inverse limit of $\left\{\left[a_{n}, b_{n}\right], f_{n}\right\}$, and every absolutely terminal subcontin- 
uum of $M$ with interior is decomposable. If $\left(\zeta_{1}, \omega_{1}\right),\left(\zeta_{2}, \omega_{2}\right), \ldots$ is a sequence of points in $M \times M$ with the property that both $\pi_{n}\left(\zeta_{n}\right)=a_{n}$ and $\pi_{n}\left(\omega_{n}\right)=b_{n}$ for each positive integer $n$ and $(z, w)$ is a subsequential limit of $\left(\zeta_{1}, \omega_{1}\right),\left(\zeta_{2}, \omega_{2}\right), \ldots$, then $\overline{z w}=M$.

Pr o of. This follows from Theorems 1 and 11.

THEOREM 12. Suppose $M$ is a semiatriodic unicoherent continuum, and suppose $X$ is a proper subcontinuum of $M$. The following are equivalent:

(1) No proper subcontinuum of $M$ contains $X$ in its interior.

(2) Every point of $M-X$ belongs to an indecomposable absolutely terminal subcontinuum of $M$ that has interior and contains a point of $X$.

(3) Every subcontinuum of $M$ with interior contains a point of $X$.

Proof. If $X$ has interior, (1), (2), and (3) are equivalent by Theorem 9; hence, it is assumed that $X$ is nowhere dense.

By Theorem 8, (1) implies (2).

Suppose (2) holds. By Corollary 7.1, $M$ can have at most two indecomposable absolutely terminal subcontinua with interior.

CASE (i). If $M$ has only one indecomposable absolutely terminal subcontinuum $Q$ with interior, then $Q=M$ by (2) and the assumption that $X$ is nowhere dense. Therefore, the only subcontinuum of $M$ with interior is $M$. It follows that every subcontinuum of $M$ with interior intersects $X$.

CASE (ii). If $M$ has two indecomposable absolutely terminal subcontinua with interior $P$ and $Q$, then $M=P \cup Q$ by (2) and the assumption that $X$ is nowhere dense. Note that each of $P$ and $Q$ contains a point not in the other since each is indecomposable and has interior. Then each of $P$ and $Q$ intersects $X$ by (2). Let $Y$ be a subcontinuum of $M$ with interior. Then $\operatorname{int}(Y)$ intersects one of $\operatorname{int}(P)$ and $\operatorname{int}(Q)$; otherwise, $\operatorname{int}(Y)$ would be a subset of a nowhere dense subset of $M$. If $\operatorname{int}(Y)$ intersects $\operatorname{int}(Q)$, then by Theorem 2, $Y \cap Q$ is a subcontinuum of $Q$ with interior. It follows that $Y$ contains $Q$ and, hence, that $Y$ intersects $X$. If $\operatorname{int}(Y)$ intersects $\operatorname{int}(P)$, the proof that $Y$ intersects $X$ is the same.

That (3) implies (1) follows from the proof in Theorem 9 that (4) implies (1), which does not require that $X$ have interior.

ExAmple. The statements of Theorems 9 and 12 do not remain true if "semiatriodic and unicoherent continuum" is replaced by "unicoherent continuum that is not a triod". This is evident from the following example. Let $M$ be a continuum that is the union of two Brouwer-Janiszewski-Knaster continua, $M_{1}$ and $M_{2}$, whose intersection is a single point different from the endpoint $M_{1}$. Note that $M_{2}$ is not an absolutely terminal subcontinuum for $M$. 
It seems likely that the statements Theorems 8, 10, and 11 would remain true if formulated for a class of continua more general than that of semiatriodic and unicoherent continua. While the statements of Theorems 5, 6, 7, 9, and 12 do not remain true when formulated for unicoherent continua that are not triods, it seems likely that they may upon replacing "absolutely terminal subcontinuum" with a more general type of subcontinuum that lies "at an end" of a continuum. Many such nonseparating subcontinua are investigated in $[1]$.

4. Points of irreducibility - part II. Throughout Section 4, the following notation is used: $M$ denotes the inverse limit of an inverse sequence of the form $\left\{\left[a_{n}, b_{n}\right], f_{n}\right\}$ where each $f_{n}$ is surjective, and $z$ and $w$ are points of $M$ that satisfy the hypothesis of Theorem 1 -which is to say, $(z, w)$ is a point of $M \times M$ to which some subsequence of $\left(\zeta_{1}, \omega_{1}\right),\left(\zeta_{2}, \omega_{2}\right), \ldots$ converges where, for each $n, \zeta_{n}$ and $\omega_{n}$ are points of $M$ such that $\pi_{n}\left(\zeta_{n}\right)=a_{n}$ and $\pi_{n}\left(\omega_{n}\right)=b_{n}$.

Theorems 1 and 12 together yield that every point of $M$ that does not belong to $\overline{z w}$ belongs to an indecomposable absolutely terminal subcontinuum of $M$ with interior. In this section, two points are constructed that have the property that the continuum irreducible between them contains every indecomposable absolutely terminal subcontinuum of $M$ with interior that is not contained by $\overline{z w}$. Furthermore, if $M$ is indecomposable, then they are points of irreducibility for $M$. It follows that $M$ is irreducible about the union of these two points with $\{z, w\}$. Some two of the four are points of irreducibility for $M$; the proof of Theorem 19 gives a procedure for selecting the two.

\subsection{Indecomposable absolutely terminal subcontinua with interior}

Definition. Suppose $f:[a, b] \rightarrow\left[a_{0}, b_{0}\right]$ is a continuous surjection, $x$ and $y$ are distinct points of $[a, b], \gamma=\min \{x, y\}, \delta=\max \{x, y\}, \gamma_{0}=$ $\min \{f(x), f(y)\}$, and $\delta_{0}=\max \{f(x), f(y)\}$. The pair $\langle x, y\rangle$ is said to be a:

- type 1 pair with respect to $f$ provided $f[[a, \gamma] \cup[\delta, b]]=\left[a_{0}, \gamma_{0}\right] \cup\left[\delta_{0}, b_{0}\right]$,

- type 2a pair with respect to $f$ provided $f[a, \gamma]$ is either $\left[a_{0}, \gamma_{0}\right]$ or $\left[\delta_{0}, b_{0}\right]$, and $f[a, \delta]=\left[a_{0}, b_{0}\right]$,

- type $2 \mathrm{~b}$ pair with respect to $f$ provided $f[\delta, b]$ is either $\left[a_{0}, \gamma_{0}\right]$ or $\left[\delta_{0}, b_{0}\right]$, and $f[\gamma, b]=\left[a_{0}, b_{0}\right]$,

- type 3 pair with respect to $f$ provided $f[\gamma, \delta]=\left[a_{0}, b_{0}\right]$.

Theorem 13 below gives a simple procedure for constructing a pair of type 1 , type 2 , or type 3 with respect to a continuous surjection $f$ from one interval to another. Figure 2 shows the graphs of three functions with respect to which $\langle\gamma, \delta\rangle$ is a pair of type 1 , type 2 (specifically $2 \mathrm{a}$ ), and type 3 respectively. 

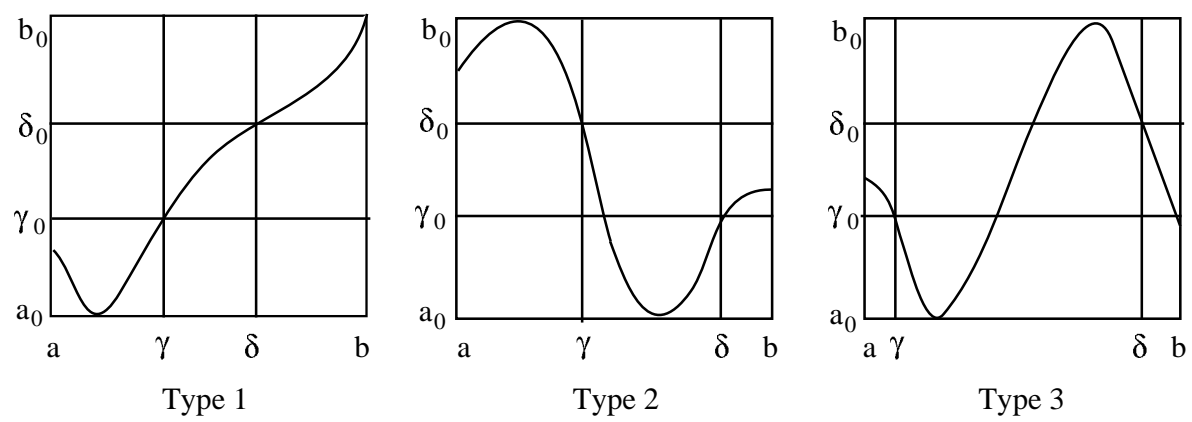

Fig. 2. Examples of type 1, type 2, and type 3 pairs

The behavior of a function having a pair of type 1, type 2 , or type 3 is reflected by its graph. For an example, denote by $f$ the function whose graph appears in Figure 2, type 1. The graph of $f$ to the left of $\gamma$ is restricted to the "lower left hand corner" of the graph of $f$, and the graph of $f$ to the right of $\delta$ is restricted to the "upper right hand corner." If, instead, the graph of $f$ to the left of $\gamma$ were restricted to the "upper left hand corner" and the graph to the right of $\delta$ were restricted to the "lower right hand corner," $\langle\gamma, \delta\rangle$ would still be a type 1 pair with respect to $f$. Note that in Figure 2, type 2 , the graph of the function to the left of $\gamma$ is restricted to the "upper left hand corner," and the graph of the function to the left of $\delta$ sweeps out the function's entire range. In particular, $\langle\gamma, \delta\rangle$ is a type 2 a pair for this function. Also note that in Figure 2, type 3, the graph of the function sweeps out the function's entire range between $\gamma$ and $\delta$.

THEOREM 13. If $f:[a, b] \rightarrow\left[a_{0}, b_{0}\right]$ is a continuous surjection, $x_{0}$ and $y_{0}$ are distinct points of $\left[a_{0}, b_{0}\right], x^{\prime}=\min \left\{x \in[a, b]: f(x)=x_{0}\right\}$, $y^{\prime}=\max \left\{x \in[a, b]: f(x)=y_{0}\right\}, x^{\prime \prime}=\max \left\{x \in[a, b]: f(x)=x_{0}\right\}$, and $y^{\prime \prime}=\min \left\{x \in[a, b]: f(x)=y_{0}\right\}$, then one of $\left\langle x^{\prime}, y^{\prime}\right\rangle$ and $\left\langle x^{\prime \prime}, y^{\prime \prime}\right\rangle$ is of type 1 , type 2 , or type 3 with respect to $f$.

Proof. Let $\gamma^{\prime}=\min \left\{x^{\prime}, y^{\prime}\right\}, \delta^{\prime}=\max \left\{x^{\prime}, y^{\prime}\right\}, \gamma^{\prime \prime}=\min \left\{x^{\prime \prime}, y^{\prime \prime}\right\}$, and $\delta^{\prime \prime}=\max \left\{x^{\prime \prime}, y^{\prime \prime}\right\}$. Suppose $\left\langle x^{\prime}, y^{\prime}\right\rangle$ is of neither type 1, type 2, nor type 3 with respect to $f$. Since it is not of type 3 , one of $f\left[a, \gamma^{\prime}\right]$ and $f\left[\delta^{\prime}, b\right]$ must intersect $\left\{a_{0}, b_{0}\right\}$; and since it is not of type 1 , one must intersect $\left(\gamma_{0}, \delta_{0}\right)$. Suppose that neither $f\left[a, \gamma^{\prime}\right]$ nor $f\left[\delta^{\prime}, b\right]$ intersects both $\left\{a_{0}, b_{0}\right\}$ and $\left(\gamma_{0}, \delta_{0}\right)$. Then one must be a subset of $\left(a_{0}, b_{0}\right)$, and the other either $\left[a_{0}, \gamma_{0}\right]$ or $\left[\delta_{0}, b_{0}\right]$. Consequently, $\left\langle x^{\prime}, y^{\prime}\right\rangle$ is a type 2 pair, which is a contradiction. It is assumed that $f\left[a, \gamma^{\prime}\right]$ intersects both $\left\{b_{0}\right\}$ and $\left(\gamma_{0}, \delta_{0}\right)$; the proof is similar in case it intersects $\left\{a_{0}\right\}$ and $\left(\gamma_{0}, \delta_{0}\right)$, or in case $f\left[\delta^{\prime}, b\right]$ intersects both $\left\{a_{0}, b_{0}\right\}$ and $\left(\gamma_{0}, \delta_{0}\right)$. Then $\delta_{0} \in f\left[a, \gamma^{\prime}\right)$. Since $x_{0}$ cannot be in $f\left[a, x^{\prime}\right)$, which contains $f\left[a, \gamma^{\prime}\right)$, it follows that $\delta_{0}=y_{0}$ and, hence, that $y^{\prime \prime}<\gamma^{\prime}$. Since $\gamma^{\prime} \leq x^{\prime} \leq x^{\prime \prime}$, this gives $\gamma^{\prime \prime}=y^{\prime \prime}$ and $\delta^{\prime \prime}=x^{\prime \prime}$. Note that if $b_{0} \in f\left[a, y^{\prime \prime}\right]$, then $f\left[a, y^{\prime \prime}\right]=\left[\delta_{0}, b_{0}\right]$ 
since $\delta_{0}=y_{0}$ and $y_{0} \notin f\left[a, y^{\prime \prime}\right)$. If $a_{0} \notin f\left[x^{\prime \prime}, b\right]$, then $\left\langle x^{\prime \prime}, y^{\prime \prime}\right\rangle$ is a type 2a pair if $b_{0} \in f\left[a, y^{\prime \prime}\right]$, and a type 3 pair otherwise. If $a_{0} \in f\left[x^{\prime \prime}, b\right]$, then $f\left[x^{\prime \prime}, b\right]=\left[a_{0}, x_{0}\right]=\left[a_{0}, \gamma_{0}\right]$; so $\left\langle x^{\prime \prime}, y^{\prime \prime}\right\rangle$ is a type 1 pair if $b_{0} \in f\left[a, y^{\prime \prime}\right]$, and a type $2 \mathrm{~b}$ pair otherwise.

Lemma 2. If $A$ is an absolutely terminal subcontinuum of $M$ with interior, then there exists a positive integer, $N$, such that if $n$ is a positive integer not less than $N$, then $\pi_{n}(A)$ intersects $\left\{a_{n}, b_{n}\right\}$.

Proof. Suppose $A$ is a subcontinuum of $M$ with interior that has the property that $\pi_{n}(A)$ misses $\left\{a_{n}, b_{n}\right\}$ for infinitely many positive integers $n$. Then $\pi_{n}(M-A)$ contains both $a_{n}$ and $b_{n}$ for infinitely many positive integers $n$. It follows that $\overline{M-A}$ is not connected; otherwise, $\pi_{n}[\overline{M-A}]$ would contain $\left[a_{n}, b_{n}\right]$ for infinitely many positive integers $n$, from which it would follow that $\overline{M-A}=M$ in contradiction with the hypothesis that $A$ has interior. Consequently, $A$ is not an absolutely terminal subcontinuum of $M$ by Theorem 5 .

LEMMA 3. Suppose $x$ and $y$ are distinct points of $M$ such that $\left\langle x_{n+1}, y_{n+1}\right\rangle$ is a pair of type 1 or type 2 with respect to $f_{n}$ for all but finitely many positive integers $n$, and a pair of type 2 with respect to $f_{n}$ for infinitely many positive integers $n$. If $\overline{x y}$ is a proper subcontinuum of $M$, then there are a positive integer $N$ and a sequence $A_{1}, A_{2}, \ldots$ of sets where $A_{n}$ is one of $\left[a_{n}, \min \left\{x_{n}, y_{n}\right\}\right]$ and $\left[\max \left\{x_{n}, y_{n}\right\}, b_{n}\right]$ for $n$ not less than $N$ with the property that $f_{n}\left[A_{n+1}\right]=A_{n}$ for each $n$, and $M-\overline{x y} \subset \lim \left\{A_{n}, f_{n} \mid A_{n+1}\right\}$.

Proof. There is a positive integer $N$ such that $\left\langle x_{n+1}, y_{n+1}\right\rangle$ is a pair of type 1 or type 2 with respect to $f_{n}$ and $\pi_{n}[\overline{x y}]$ does not contain both $a_{n}$ and $b_{n}$ for each integer $n$ not less than $N$. Since $\left\langle x_{n+1}, y_{n+1}\right\rangle$ is a type 2 pair for infinitely many positive integers $n, \pi_{n}[\overline{x y}]$ intersects $\left\{a_{n}, b_{n}\right\}$ for infinitely many positive integers $n$. Note that if $\pi_{k+1}[\overline{x y}]$ intersects $\left\{a_{k+1}, b_{k+1}\right\}$, and $\left\langle x_{k+1}, y_{k+1}\right\rangle$ is a pair of type 1 or type 2 with respect to $f_{k}$, then $\pi_{k}[\overline{x y}]$ intersects $\left\{a_{k}, b_{k}\right\}$. Since $\pi_{n}[\overline{x y}]$ intersects $\left\{a_{n}, b_{n}\right\}$ for infinitely many positive integers $n$, and $\left\langle x_{n+1}, y_{n+1}\right\rangle$ is a pair of type 1 or type 2 for $n$ not less than $N$, it follows that $\pi_{n}[\overline{x y}]$ intersects $\left\{a_{n}, b_{n}\right\}$ for $n$ not less than $N$. For these values of $n$, denote $\min \left\{x_{n}, y_{n}\right\}$ and $\max \left\{x_{n}, y_{n}\right\}$ by $\gamma_{n}$ and $\delta_{n}$ respectively, and let $A_{n}$ be $\left[a_{n}, \gamma_{n}\right]$ if $a_{n} \notin \pi_{n}[\overline{x y}]$ and $\left[\delta_{n}, b_{n}\right]$ if $b_{n} \notin \pi_{n}[\overline{x y}]$. For positive integers $n$ less than $N$, denote $f_{n}^{N}\left[A_{N}\right]$ by $A_{n}$. Observe that $A_{n}$ contains $\left[a_{n}, b_{n}\right]-\pi_{n}[\overline{x y}]$ for each positive integer $n$.

Let $k$ be a given positive integer. It is shown that $f_{k}\left[A_{k+1}\right]=A_{k}$. If $k$ is less than $N$, then $f_{k}\left[A_{k+1}\right]=f_{k}\left[f_{k+1}^{N}\left[A_{N}\right]\right]=f_{k}^{N}\left[A_{N}\right]=A_{k}$. Suppose $k$ is not less than $N$.

CASE (i). Suppose $\left\langle x_{k+1}, y_{k+1}\right\rangle$ is a type 1 pair. Either $f_{k}\left[A_{k+1}\right]=\left[a_{k}, \gamma_{k}\right]$ or $f_{k}\left[A_{k+1}\right]=\left[\delta_{k}, b_{k}\right]$. If $f_{k}\left[A_{k+1}\right]=\left[a_{k}, \gamma_{k}\right]$, then $b_{k} \in f_{k}\left[\left[a_{k+1}, b_{k+1}\right]-\right.$ 
$\left.A_{k+1}\right] \subset f_{k}\left[\pi_{k+1}[\overline{x y}]\right]=\pi_{k}[\overline{x y}]$. Hence, $a_{k} \notin \pi_{k}[\overline{x y}]$, so by definition, $A_{k}$ is $\left[a_{k}, \gamma_{k}\right]$. It follows that $f_{k}\left[A_{k+1}\right]=A_{k}$. Similarly, if $f_{k}\left[A_{k+1}\right]=\left[\delta_{k}, b_{k}\right]$, then $f_{k}\left[A_{k+1}\right]=A_{k}$.

CASE (ii). Suppose $\left\langle x_{k+1}, y_{k+1}\right\rangle$ is a type 2 pair. It is assumed that $\left\langle x_{k+1}, y_{k+1}\right\rangle$ is a type 2 a pair; the proof is similar if it is a type $2 \mathrm{~b}$ pair. Since $f_{k}\left[a_{k+1}, \delta_{k+1}\right]$ is all of $\left[a_{k}, b_{k}\right], a_{k+1}$ is not in $\pi_{k+1}[\overline{x y}]$. Thus, $A_{k+1}=$ $\left[a_{k+1}, \gamma_{k+1}\right]$. If $f_{k}\left[A_{k+1}\right]=\left[a_{k}, \gamma_{k}\right]$, then $f_{k}\left[\pi_{k+1}[\overline{x y}]\right]=\pi_{k}[\overline{x y}]$ must contain $b_{k}$. Therefore, by the definition of $A_{k}, A_{k}=\left[a_{k}, \gamma_{k}\right]$. It follows that $f_{k}\left[A_{k+1}\right]=A_{k}$. Similarly, if $f_{k}\left[A_{k+1}\right]=\left[\delta_{k}, b_{k}\right]$, then $f_{k}\left[A_{k+1}\right]=A_{k}$.

Suppose $p$ is a point of $M-\overline{x y}$. Then $\pi_{n}(p)$ is in $\pi_{n}[\overline{x y}]$ for at most finitely many positive integers $n$. Consequently, $\pi_{n}(p) \in A_{n}$ for all but at most finitely many positive integers $n$ and, hence, for all $n$. It follows that $p \in \lim _{\longleftarrow}\left\{A_{n}, f_{n+1} \mid A_{n+1}\right\}$.

TheOREM 14. Suppose $Q$ is an indecomposable absolutely terminal subcontinuum of $M$ with interior. If $x$ and $y$ are distinct points of $M$ such that $\left\langle x_{n+1}, y_{n+1}\right\rangle$ is a pair of type 1, type 2 , or type 3 with respect to $f_{n}$ for all but finitely many integers $n$, and $\operatorname{int}\left(\pi_{n}[Q]\right)$ contains $x_{n}$ (or $\left.y_{n}\right)$ for infinitely many positive integers $n$, then $\overline{x y}$ contains $Q$.

Proof. If $\overline{x y}=M$, then $\overline{x y}$ certainly contains $Q$. Hence, it is assumed that $\overline{x y}$ is a proper subcontinuum of $M$. Then $\pi_{n}[\overline{x y}]=\left[a_{n}, b_{n}\right]$ for at most finitely many $n$. For each positive integer $n$, denote $\min \left\{x_{n}, y_{n}\right\}$ and $\max \left\{x_{n}, y_{n}\right\}$ by $\gamma_{n}$ and $\delta_{n}$ respectively. Since $\pi_{n}[\overline{x y}]=f_{n}\left[\pi_{n+1}[\overline{x y}]\right]$ for each $n$, and $f_{n}\left[\pi_{n+1}[\overline{x y}]\right]$ contains $f_{n}\left[\gamma_{n+1}, \delta_{n+1}\right]$ for each $n$, it follows that $\pi_{n}[\overline{x y}]$ contains $f_{n}\left[\gamma_{n+1}, \delta_{n+1}\right]$ for each $n$. Therefore, $\left\langle x_{n+1}, y_{n+1}\right\rangle$ is a type 3 pair for at most finitely many positive integers $n$.

CASE (i). Suppose $\left\langle x_{n+1}, y_{n+1}\right\rangle$ is a type 2 pair for at most finitely many $n$. There is a positive integer $N$ such that $\left\langle x_{n+1}, y_{n+1}\right\rangle$ is a type 1 pair for each integer $n$ not less than $N$ which, by Lemma 2, can be chosen large enough to guarantee that $\pi_{n}[Q]$ intersects $\left\{a_{n}, b_{n}\right\}$ for each integer $n$ not less than $N$. Let $B_{n}$ be $\left[a_{n}, \gamma_{n}\right] \cup\left[\delta_{n}, b_{n}\right]$ for $n$ not less than $N$, and let $B_{n}$ be $f_{n}^{N}\left[B_{N}\right]$ for $n$ less than $N$. For $n$ not less than $N,\left\langle x_{n+1}, y_{n+1}\right\rangle$ is a type 1 pair, so $f_{n}\left[B_{n+1}\right]=B_{n}$. If $n$ is less than $N$, then $f_{n}\left[B_{n+1}\right]=f_{n}\left[f_{n+1}^{N}\left[B_{N}\right]\right]=$ $f_{n}^{N}\left[B_{N}\right]=B_{n}$. Let $B=\lim _{\longleftarrow}\left\{B_{n}, f_{n} \mid B_{n+1}\right\}$. If $p$ is a point of $M-\overline{x y}$, then $\pi_{n}(p)$ is in $\pi_{n}[\overline{x y}]$ for at most finitely many positive integers $n$. Since $\pi_{n}[\overline{x y}]$ contains $\left[\gamma_{n}, \delta_{n}\right]$ for each positive integer $n$ it follows that $\pi_{n}(p)$ is in $\left[a_{n}, \gamma_{n}\right] \cup\left[\delta_{n}, b_{n}\right]$ for infinitely many integers $n$ and, therefore, in $B_{n}$ for all positive integers $n$. Consequently, $p \in B$ and $M-\overline{x y} \subset B$. It follows that $\overline{x y}$ contains $B^{\mathrm{c}}$ which, in turn, contains $B^{\mathrm{c}} \cap Q$. If $Q$ does not intersect $B$, then the conclusion of the theorem follows. Suppose $Q$ does intersect $B$. Denote $\pi_{n}[Q]$ by $Q_{n}$. Then $Q \cap B=\lim _{\longleftarrow}\left\{Q_{n} \cap B_{n}, f_{n} \mid Q_{n+1} \cap B_{n+1}\right\}$. Since, 
for $n$ not less than $N, B_{n}=\left[a_{n}, \gamma_{n}\right] \cup\left[\delta_{n}, b_{n}\right]$, and $Q_{n}$ is a subinterval of $\left[a_{n}, b_{n}\right]$ that intersects $\left\{a_{n}, b_{n}\right\}$, it follows that either $Q_{n} \cap B_{n}$ is an interval for $n$ not less than $N$ or there is a positive integer $N_{1}$ such that $Q_{n} \cap B_{n}$ is the union of two disjoint intervals for $n$ not less than $N_{1}$. In the latter case, it follows that $Q \cap B$ is the union of two proper subcontinua of $Q$; hence, $Q \cap B$ is nowhere dense in $Q$. In the former case, since $x_{n}$ is a boundary point of $B_{n}$ for $n$ not less than $N$, and $\operatorname{int}\left(Q_{n}\right)$ contains $x_{n}$ for infinitely many integers $n$, it follows that $Q_{n} \cap B_{n}$ is a proper subinterval of $Q_{n}$ for infinitely many integers $n$. Hence, $Q \cap B$ is a proper subcontinuum of $Q$ and, therefore, nowhere dense in $Q$. Since $\overline{x y}$ contains $B^{\mathrm{c}} \cap Q$ and $\overline{x y}$ is closed, it follows that $\overline{x y}$ contains $Q$.

CASE (ii). Suppose $\left\langle x_{n+1}, y_{n+1}\right\rangle$ is a type 2 pair for infinitely many positive integers $n$. By Lemma 3 , there is a positive integer $N$ and a sequence $A_{1}, A_{2}, \ldots$ of sets where each $A_{n}$ is one of $\left[a_{n}, \gamma_{n}\right]$ and $\left[\delta_{n}, b_{n}\right]$ for $n$ not less than $N$ with the property that $f_{n}\left[A_{n+1}\right]=A_{n}$ for each $n$, and $M-\overline{x y} \subset$ $\lim _{\lfloor}\left\{A_{n}, f_{n} \mid A_{n+1}\right\}$; furthermore, by Lemma $2, N$ can be chosen large enough to guarantee that $\pi_{n}[Q]$ intersects $\left\{a_{n}, b_{n}\right\}$ for $n$ not less than $N$. Let $A=$ $\lim _{\{}\left\{A_{n}, f_{n} \mid A_{n+1}\right\}$. If $Q$ does not intersect $A$, then since $M-\overline{x y}$ is a subset of $A$, the conclusion of the theorem follows. Suppose $Q$ intersects $A$. Note that $Q \cap A$ is a continuum since $M$ is hereditarily unicoherent. Denote $\pi_{n}[Q]$ by $Q_{n}$. Then $Q \cap A=\varliminf_{\lfloor}\left\{Q_{n} \cap A_{n}, f_{n} \mid\left(Q_{n+1} \cap A_{n+1}\right)\right\}$. Since $A_{n}$ is one of $\left[a_{n}, \gamma_{n}\right]$ and $\left[\delta_{n}, b_{n}\right]$ for each integer $n$ not less than $N$, it follows that $x_{n}$ is not an interior point of $A_{n}$ for these values of $n$. Since $\operatorname{int}\left(\pi_{n}[Q]\right)$ contains $x_{n}$ for infinitely many positive integers $n, Q_{n}$ must contain a point that is not in $A_{n}$ for infinitely many values of $n$. It follows that $A \cap Q$ is a proper subcontinuum of $Q$ and, hence, nowhere dense in $Q$. Since $\overline{x y}$ contains $A^{\mathrm{c}} \cap Q$, it follows that $\overline{x y}$ contains $Q$.

Corollary 14.1. If $M$ is indecomposable and $x$ and $y$ are distinct points of $M$ such that $\left\langle x_{n+1}, y_{n+1}\right\rangle$ is a pair of type 1, type 2 , or type 3 with respect to $f_{n}$ for all but finitely many $n$, then $M$ is irreducible between $x$ and $y$.

Proof. Let $Q=M$ in Theorem 14.

4.2. Locating indecomposable absolutely terminal subcontinua with interior. In Section 4.1, a simple algorithm is given for generating points $x$ and $y$ of $M$ with the property that $\left\langle x_{n+1}, y_{n+1}\right\rangle$ is a pair of type 1 , type 2 , or type 3 for all or all but finitely many positive integers $n$, and Theorem 14 guarantees that $\overline{x y}$ contains an indecomposable absolutely terminal subcontinuum $Q$ of $M$ with interior if $\operatorname{int}\left(\pi_{n}[Q]\right)$ contains $x_{n}$ (or $\left.y_{n}\right)$ for infinitely many $n$. This raises the following question: how are $x$ and $y$ to be constructed in order to guarantee that for each indecomposable absolutely terminal sub- 
continuum $Q$ of $M$ with interior, $\operatorname{int}\left(\pi_{n}[Q]\right)$ contains either $x_{n}$ or $y_{n}$ for infinitely many $n$ ? In order to answer this, it is well to introduce the notion of a "type 4 pair."

Definition. Let $f:[a, b] \rightarrow\left[a_{0}, b_{0}\right]$ be a continuous surjection, $x$ and $y$ be distinct points of $[a, b], \gamma=\min \{x, y\}, \delta=\max \{x, y\}, \gamma_{0}=$ $\min \{f(x), f(y)\}$, and $\delta_{0}=\max \{f(x), f(y)\}$. The pair $\langle x, y\rangle$ is said to be a type 4 pair with respect to $f$ provided neither $f[a, \gamma)$ nor $f(\delta, b]$ intersects both $\left\{a_{0}, b_{0}\right\}$ and $\left\{\gamma_{0}, \delta_{0}\right\}$.

REMARK. If $\langle x, y\rangle$ is a type 4 pair with respect to $f$, then it must be a pair of type 1 , type 2 , or type 3 with respect to $f$. In particular, if $\langle x, y\rangle$ is a type 4 pair with respect to $f$ and:

- both $f[a, \gamma)$ and $f(\delta, b]$ intersect $\left\{a_{0}, b_{0}\right\}$, then $\langle x, y\rangle$ is a type 1 pair with respect to $f$.

- $f[a, \gamma)$ intersects $\left\{a_{0}, b_{0}\right\}$ but $f(\delta, b]$ does not, then $\langle x, y\rangle$ is a type $2 \mathrm{a}$ pair with respect to $f$.

- $f(\delta, b]$ intersects $\left\{a_{0}, b_{0}\right\}$ but $f[a, \gamma)$ does not, then $\langle x, y\rangle$ is a type $2 \mathrm{~b}$ pair with respect to $f$.

- neither $f[a, \gamma)$ nor $f(\delta, b]$ intersects $\left\{a_{0}, b_{0}\right\}$, then $\langle x, y\rangle$ is a type 3 pair with respect to $f$.

The converse, however, is not true. Let $f$ be the full-tent map - the mapping of $[0,1]$ onto itself that is linear on the intervals $[0,1 / 2]$ and $[1 / 2,1]$, and satisfies $f(0)=f(1)=0$ and $f(1 / 2)=1$. The pair $\langle .2, .7\rangle$ is a type 2a pair with respect to $f$, but not a type 4 pair.

Definition. Let $f:[a, b] \rightarrow\left[a_{0}, b_{0}\right]$ be a continuous surjection. The map $f$ is a two-pass map provided there is a point $c$ of $[a, b]$ such that $f[a, c]=$ $f[c, b]=\left[a_{0}, b_{0}\right]$.

The following theorem shows that for a continuous surjection $f$ that is not a two-pass map, the same procedure used in Theorem 13 to construct a pair of either type 1 , type 2 , or type 3 with respect to $f$ may be used to construct a type 4 pair with respect to $f$.

TheOREM 15. Suppose $f:[a, b] \rightarrow\left[a_{0}, b_{0}\right]$ is a continuous surjection that is not a two-pass map. If $x_{0}$ and $y_{0}$ are distinct points of $\left[a_{0}, b_{0}\right]$, $x^{\prime}=\min \left\{x \in[a, b]: f(x)=x_{0}\right\}, y^{\prime}=\max \left\{x \in[a, b]: f(x)=y_{0}\right\}, x^{\prime \prime}=$ $\max \left\{x \in[a, b]: f(x)=x_{0}\right\}$, and $y^{\prime \prime}=\min \left\{x \in[a, b]: f(x)=y_{0}\right\}$; then one of $\left\langle x^{\prime}, y^{\prime}\right\rangle$ and $\left\langle x^{\prime \prime}, y^{\prime \prime}\right\rangle$ is a type 4 pair with respect to $f$.

Proof. Let $\gamma_{0}=\min \left\{x_{0}, y_{0}\right\}, \delta_{0}=\max \left\{x_{0}, y_{0}\right\}, \gamma^{\prime}=\min \left\{x^{\prime}, y^{\prime}\right\}, \delta^{\prime}=$ $\max \left\{x^{\prime}, y^{\prime}\right\}, \gamma^{\prime \prime}=\min \left\{x^{\prime \prime}, y^{\prime \prime}\right\}$, and $\delta^{\prime \prime}=\max \left\{x^{\prime \prime}, y^{\prime \prime}\right\}$. Suppose $\left\langle x^{\prime}, y^{\prime}\right\rangle$ is not a type 4 pair with respect to $f$. Then one of $f\left[a, \gamma^{\prime}\right)$ and $f\left(\delta^{\prime}, b\right]$ intersects both $\left\{a_{0}, b_{0}\right\}$ and $\left\{\gamma_{0}, \delta_{0}\right\}$. It is assumed that $f\left[a, \gamma^{\prime}\right)$ intersects both $\left\{b_{0}\right\}$ 
and $\left\{\gamma_{0}, \delta_{0}\right\}$; the proof is similar in case it intersects both $\left\{a_{0}\right\}$ and $\left\{\gamma_{0}, \delta_{0}\right\}$, or in case $f\left(\delta^{\prime}, b\right]$ intersects both $\left\{a_{0}, b_{0}\right\}$ and $\left\{\gamma_{0}, \delta_{0}\right\}$. Then $\delta_{0} \in f\left[a, \gamma^{\prime}\right)$. It follows that $\delta_{0}$ cannot be $x_{0}$ since $x_{0} \notin f\left[a, x^{\prime}\right)$ and $\gamma^{\prime} \leq x^{\prime}$. Hence, $\delta_{0}=y_{0}$, and $y^{\prime \prime}<\gamma^{\prime}$. Since $\gamma^{\prime} \leq x^{\prime} \leq x^{\prime \prime}$, this gives $\gamma^{\prime \prime}=y^{\prime \prime}, \delta^{\prime \prime}=x^{\prime \prime}$, and $y^{\prime \prime} \leq x^{\prime}$. Therefore, $f\left[a, y^{\prime \prime}\right)$ does not intersect $\left\{\gamma_{0}, \delta_{0}\right\}$. Either $x^{\prime \prime}>y^{\prime}$ or $x^{\prime \prime}<y^{\prime}$. If $x^{\prime \prime}>y^{\prime}$, then $f\left(x^{\prime \prime}, b\right]$ does not intersect $\left\{\gamma_{0}, \delta_{0}\right\}$. Suppose $x^{\prime \prime}<y^{\prime}$. Neither $f\left[a, \gamma^{\prime}\right)$ nor $f\left(x^{\prime \prime}, b\right]$ contains $x_{0}$, so neither can contain both $a_{0}$ and $b_{0}$. Recall that $b_{0} \in f\left[a, \gamma^{\prime}\right)$. Therefore, $f\left(x^{\prime \prime}, b\right]$ cannot contain $b_{0}$, otherwise $f\left[\gamma^{\prime}, x^{\prime \prime}\right]$ would contain $a_{0}$ and $f$ would be a two-pass map. Hence, $b_{0} \notin f\left(x^{\prime \prime}, b\right]$. Suppose $a_{0} \in f\left(x^{\prime \prime}, b\right]$, and let $\alpha$ be the point of $\left(x^{\prime \prime}, b\right]$ whose image is $a_{0}$. Then the interval whose endpoints are $\alpha$ and $y^{\prime}$ is a subinterval of $\left(x^{\prime \prime}, b\right]$. Since $y_{0}=\delta_{0}$, it follows that $a_{0} \leq x_{0}<y_{0}$. Then the interval whose endpoints are $\alpha$ and $y^{\prime}$ and, hence, the interval $\left(x^{\prime \prime}, b\right]$ must contain a point whose image is $x_{0}$. This is a contradiction with the definition of $x^{\prime \prime}$. Therefore, $f\left(x^{\prime \prime}, b\right]$ does not intersect $\left\{a_{0}, b_{0}\right\}$.

Lemma 4. Suppose $f:[a, b] \rightarrow\left[a_{0}, b_{0}\right]$ is a continuous surjection, $\langle x, y\rangle$ is a type 4 pair with respect to $f, f$ is not a two-pass map, $J$ is a closed subinterval of $[a, b]$ that intersects $\{a, b\}$, and $J_{0}=f[J]$ intersects $\left\{a_{0}, b_{0}\right\}$. If $\operatorname{int}\left(J_{0}\right)$ intersects $\{f(x), f(y)\}$, then $\operatorname{int}(J)$ intersects $\{x, y\}$.

Proof. Let $\gamma=\min \{x, y\}, \delta=\max \{x, y\}, \gamma_{0}=\min \{f(x), f(y)\}$, and $\delta_{0}=\max \{f(x), f(y)\}$. Suppose $\operatorname{int}(J)$ does not intersect $\{x, y\}$. If $J$ is degenerate, it follows that $\operatorname{int}\left(J_{0}\right)$ does not intersect $\{f(x), f(y)\}$. Suppose $J$ is nondegenerate. Then $\overline{f[\operatorname{int}(J)]}=f[\overline{\operatorname{int}(J)}]=f[J]=J_{0}$. Since $J$ is a subinterval of $[a, b]$ that intersects $\{a, b\}$, but int $(J)$ fails to intersect $\{x, y\}$, it follows that $\operatorname{int}(J)$ must be a subset of either $[a, \gamma)$ or $(\delta, b]$. Therefore, since $\langle x, y\rangle$ is a type 4 pair, $f[\operatorname{int}(J)]$ cannot intersect both $\left\{a_{0}, b_{0}\right\}$ and $\left\{\gamma_{0}, \delta_{0}\right\}$. However, its closure, $J_{0}$, must intersect $\left\{a_{0}, b_{0}\right\}$. Consequently, $J_{0} \subset\left[a_{0}, \gamma_{0}\right] \cup\left[\delta_{0}, b_{0}\right]$. Since $\left\{\gamma_{0}, \delta_{0}\right\}=\{f(x), f(y)\}$, it follows that $\operatorname{int}\left(J_{0}\right)$ does not intersect $\{f(x), f(y)\}$.

THEOREM 16. Suppose $A$ is an absolutely terminal subcontinuum of $M$ with interior, $N$ is a positive integer such that $\pi_{n}[A]$ intersects $\left\{a_{n}, b_{n}\right\}$ for $n$ not less than $N$, and $f_{n}$ is not a two-pass map for $n$ not less than $N$. If $x$ and $y$ are points of $M$ such that $\left\langle x_{n+1}, y_{n+1}\right\rangle$ is a type 4 pair for $n$ not less than $N$, and $\operatorname{int}\left(\pi_{N}[A]\right)$ contains one of $x_{N}$ and $y_{N}$, then $\operatorname{int}\left(\pi_{n}[A]\right)$ intersects $\left\{x_{n}, y_{n}\right\}$ for $n$ not less than $N$.

Proof. Since $\pi_{n}[A]=f_{n}\left[\pi_{n+1}[A]\right]$, Lemma 4 entails that $\operatorname{int}\left(\pi_{N+1}[A]\right)$ intersects $\left\{x_{N+1}, y_{N+1}\right\}$. Proceeding inductively yields the desired result.

Recall the question posed at the beginning of this section: how are $x$ and $y$ to be constructed in order to guarantee that for each indecomposable absolutely terminal subcontinuum $Q$ of $M$ with interior, $\operatorname{int}\left(\pi_{n}[Q]\right)$ con- 
tains either $x_{n}$ or $y_{n}$ for infinitely many $n$ ? For an inverse sequence with finitely many two-pass maps, Theorem 16 reduces the above question to the following.

1. How is $N$ to be chosen in order to guarantee that if $Q$ is an indecomposable absolutely terminal subcontinuum of $M$ with interior, then $\pi_{n}[Q]$ intersects $\left\{a_{n}, b_{n}\right\}$ for $n$ not less than $N$ ?

2. How are $x_{N}$ and $y_{N}$ to be defined in order to guarantee that if $Q$ is an indecomposable absolutely terminal subcontinuum of $M$ with interior, then $\operatorname{int}\left(\pi_{N}[Q]\right)$ contains one of $x_{N}$ and $y_{N}$ ?

To answer these, it will be useful to consider the points $z$ and $w$ (defined in the first paragraph of Section 4).

Lemma 5. If $A$ is an absolutely terminal subcontinuum of $M$ with interior, then $A$ contains one of $z$ and $w$.

Proof. There is a subsequence, $\left(\zeta_{n_{1}}, \omega_{n_{1}}\right),\left(\zeta_{n_{2}}, \omega_{n_{2}}\right), \ldots$, of $\left(\zeta_{1}, \omega_{1}\right)$, $\left(\zeta_{2}, \omega_{2}\right), \ldots$ that converges to $(z, w)$. For each positive integer $k, \pi_{n_{k}}\left(\zeta_{n_{k}}\right)=$ $a_{n_{k}}$ and $\pi_{n_{k}}\left(\omega_{n_{k}}\right)=b_{n_{k}}$. Then by Lemma $2, \pi_{n_{k}}[A]$ contains $\pi_{n_{k}}\left(\zeta_{n_{k}}\right)$ for infinitely many positive integers $k$, or $\pi_{n_{k}}[A]$ contains $\pi_{n_{k}}\left(\omega_{n_{k}}\right)$ for infinitely many positive integers $k$. Suppose the former, and let $m$ be a given positive integer. Note that $\pi_{m}[A]=f_{m}^{n_{k}}\left[\pi_{n_{k}}[A]\right]$ and $\pi_{m}\left(\zeta_{n_{k}}\right)=f_{m}^{n_{k}}\left(\pi_{n_{k}}\left(\zeta_{n_{k}}\right)\right)$ for $n_{k}$ not less than $m$. Then $\pi_{m}[A]$ contains $\pi_{m}\left(\zeta_{n_{k}}\right)$ for infinitely many positive integers $k$. Since $\pi_{m}\left[\zeta_{n_{1}}\right], \pi_{m}\left[\zeta_{n_{2}}\right], \ldots$ converges to $\pi_{m}[z]$ and $\pi_{m}[A]$ is compact, it follows that $\pi_{m}[A]$ contains $\pi_{m}(z)$. Hence, $A$ contains $z$. Similarly, if $\pi_{n_{k}}[A]$ contains $\pi_{n_{k}}\left(\omega_{n_{k}}\right)$ for infinitely many positive integers $k$, then $A$ contains $w$.

Definitions. An E-sequence of $M$ with respect to the points $z$ and $w$ is a sequence $E_{N}, E_{N+1}, \ldots$ with each $E_{n}$ nondegenerate and equal to either $\left[a_{n}, \min \left\{z_{n}, w_{n}\right\}\right]$ or $\left[\max \left\{z_{n}, w_{n}\right\}, b_{n}\right]$. It is called imagewise nondecreasing provided $E_{n} \subset f_{n}\left[E_{n+1}\right]$ for each $n$. An E-sequence $\widetilde{E}_{N}, \widetilde{E}_{N+1}, \ldots$ for which $\widetilde{E}_{n}$ is $\left[a_{n}, \min \left\{z_{n}, w_{n}\right\}\right]$ if $E_{n}=\left[\max \left\{z_{n}, w_{n}\right\}, b_{n}\right]$ and $\left[\max \left\{z_{n}, w_{n}\right\}, b_{n}\right]$ otherwise is the conjugate E-sequence of $E_{N}, E_{N+1}, \ldots$

Lemma 6. If $Q$ is an indecomposable absolutely terminal subcontinuum of $M$ with interior that is not contained by $\overline{z w}$, then there exists an imagewise nondecreasing E-sequence $E_{N}, E_{N+1}, \ldots$ such that $E_{n} \subset \pi_{n}[Q]$ for each $n$.

Pro of. Let $Q$ be an indecomposable absolutely terminal subcontinuum of $M$ with interior that is not contained by $\overline{z w}$, and let $P=\overline{z w} \cup \overline{M-Q}$. If $Q=M$, then $P$ is the continuum $\overline{z w}$. If $Q$ is not all of $M$, then $\overline{M-Q}$ is a continuum by Theorem 5 , and $\overline{M-Q}$ intersects $\overline{z w}$ by (1) and (3) of Theorem 12. Hence, $P$ is a continuum. Since $Q$ also has interior, $Q$ intersects 
$\overline{z w}$. The continuum $M$ is hereditarily unicoherent so $\overline{z w} \cap Q$ is a continuum. By assumption, $\overline{z w}$ does not contain $Q$; therefore, $\overline{z w} \cap Q$ is a proper subcontinuum of $Q$. Thus $\overline{z w} \cap Q$ is nowhere dense. The continuum $\overline{M-Q} \cap Q$ is also nowhere dense, so $P \cap Q$ is nowhere dense. Since $Q$ has interior, it follows that $P$ does not contain $Q$. Then $P$ is a proper subcontinuum of $M$, so there is a positive integer $N$ such that $\pi_{n}[P]$ does not contain both $a_{n}$ and $b_{n}$ whenever $n$ is an integer not less than $N$.

Let $e_{N}$ be the point of $\left\{a_{N}, b_{N}\right\}$ not in $\pi_{N}[P]$, and let $E_{N}$ be $\left[a_{N}\right.$, $\left.\min \left\{z_{N}, w_{N}\right\}\right]$ if $e_{N}=a_{N}$ and $\left[\max \left\{z_{N}, w_{N}\right\}, b_{N}\right]$ if $e_{N}=b_{N}$. By Lemma 5, $\pi_{N}[Q]$ contains one of $z_{N}$ and $w_{N}$, and $e_{N} \in \pi_{N}[Q]$ since $e_{N} \notin \pi_{N}[P]$; hence, $E_{N} \subset \pi_{N}[Q]$. Note that $e_{N}$ is not in $f_{N}\left[\pi_{N+1}[P]\right]$; otherwise, it would be in $\pi_{N}[P]$. It follows that $e_{N}$ is not in $f_{N}\left[\min \left\{z_{N+1}, w_{N+1}\right\}, \max \left\{z_{N+1}, w_{N+1}\right\}\right]$ since $\overline{z w} \subset P$. If $e_{N}$ is in $f_{N}\left[a_{N+1}, \min \left\{z_{N+1}, w_{N+1}\right\}\right]$, let $e_{N+1}$ be $a_{N+1}$ and $E_{N+1}$ be $\left[a_{N+1}, \min \left\{z_{N+1}, w_{N+1}\right\}\right]$; otherwise, let $e_{N+1}$ be $b_{N+1}$ and $E_{N+1}$ be $\left[\max \left\{z_{N+1}, w_{N+1}\right\}, b_{N+1}\right]$. Then $f_{N}\left[E_{N+1}\right]$ contains both $e_{N}$ and one of $z_{N}$ and $w_{N}$. It follows that $E_{N} \subset f_{N}\left[E_{N+1}\right]$. By Lemma $5, \pi_{N+1}[Q]$ contains one of $z_{N+1}$ and $w_{N+1}$. Hence, it follows that $E_{N+1} \subset \pi_{N+1}[Q]$ if $e_{N+1} \in \pi_{N+1}[Q]$. Suppose, on the contrary, that $e_{N+1} \notin \pi_{N+1}[Q]$. Then $e_{N+1} \in \pi_{N+1}[P]$ and, hence, $E_{N+1} \subset \pi_{N+1}[P]$. Since $E_{N} \subset f_{N}\left[E_{N+1}\right]$ and $f_{N}\left[\pi_{N+1}[P]\right]=\pi_{N}[P]$, it follows that $E_{N} \subset \pi_{N}[P]$, which is not true. Consequently, $E_{N+1} \subset \pi_{N+1}[Q]$. Proceeding inductively yields a sequence $E_{N}$, $E_{N+1}, \ldots$ with the desired property.

THEOREM 17. If $M$ has no imagewise nondecreasing E-sequence with respect to $z$ and $w$, then $M$ is irreducible between $z$ and $w$.

Proof. If $M$ is not irreducible between $z$ and $w$, then by Theorems 1 and 12 , there is an indecomposable absolutely terminal subcontinuum of $M$ with interior that is not contained by $\overline{z w}$. It follows from Lemma 6 that $M$ has an imagewise nondecreasing E-sequence with respect to $z$ and $w$.

Definition. Suppose $X$ is a proper subcontinuum of $M$ such that no proper subcontinuum of $M$ contains $X$ in its interior. The integer $N$ is said to be an $E$-number for $X$ if and only if it is true that if $Q$ is an indecomposable absolutely terminal subcontinuum of $M$ with interior that is not contained by $X$, then $\pi_{n}[Q]$ intersects $\left\{a_{n}, b_{n}\right\}$ whenever $n$ is an integer not less than $N$.

THEOREM 18. Suppose $E_{N}, E_{N+1}, \ldots$ is an imagewise nondecreasing $E$ sequence with respect to $z$ and $w$.

(1) If for some positive integer $K$ not less than $N$, the sequence $\widetilde{E}_{K}$, $\widetilde{E}_{K+1}, \ldots$ is imagewise nondecreasing, then $K$ is an E-number for $\overline{z w}$.

(2) If for each positive integer $K$ not less than $N$, the sequence $\widetilde{E}_{K}$, $\widetilde{E}_{K+1}, \ldots$ is not imagewise nondecreasing, then $N$ is an E-number for $\overline{z w}$. 
Proof. If $\overline{z w}=M$, then every positive integer is trivially an E-number; hence it assumed that $\overline{z w}$ is not all of $M$. Suppose $K$ is a positive integer such that $\widetilde{E}_{n} \subset f_{n}\left[\widetilde{E}_{n+1}\right]$ for $n$ not less than $K$, and suppose $Q$ is an indecomposable absolutely terminal subcontinuum of $M$ with interior that is not contained in $\overline{z w}$. By Lemma $2, \pi_{n}[Q]$ must intersect $\left\{a_{n}, b_{n}\right\}$ for all but finitely many integers $n$; and by Lemma $5, \pi_{n}[Q]$ must intersect $\left\{z_{n}, w_{n}\right\}$ for each positive integer $n$. It follows that for all but finitely many integers $n$, $\pi_{n}[Q]$ contains one of $E_{n}$ and $\widetilde{E}_{n}$. Suppose $\pi_{n}[Q]$ contains $E_{n}$ for infinitely many integers $n$. Then for $n$ not less than $N$, there is an integer $m$ not less than $n$ such that $E_{m} \subset \pi_{m}[Q]$. Since $E_{N}, E_{N+1}, \ldots$ is imagewise nondecreasing, $E_{n} \subset f_{n}^{m}\left[E_{m}\right]$. Hence, $E_{n} \subset f_{n}^{m}\left[\pi_{m}[Q]\right]=\pi_{n}[Q]$ for $n$ not less than $N$. It follows that $\pi_{n}[Q]$ intersects $\left\{a_{n}, b_{n}\right\}$ for $n$ not less than $N$. Similarly, if $\pi_{n}[Q]$ contains $\widetilde{E}_{n}$ for infinitely many integers $n$, then $\pi_{n}[Q]$ intersects $\left\{a_{n}, b_{n}\right\}$ for $n$ not less than $K$. In either case, $\pi_{n}[Q]$ intersects $\left\{a_{n}, b_{n}\right\}$ for $n$ not less than $K$. It follows that $K$ is an E-number.

Suppose $\widetilde{E}_{n} \not \subset f_{n}\left[\widetilde{E}_{n+1}\right]$ for infinitely many integers $n$, and suppose $Q$ is an indecomposable absolutely terminal subcontinuum of $M$ with interior that is not contained in $\overline{z w}$. By Lemma 6 , there is a sequence $I_{1}, I_{2}, \ldots$ where each $I_{n}$ is one of $E_{n}$ and $\widetilde{E}_{n}$ with the property that both $I_{n} \subset f_{n}\left[I_{n+1}\right]$ and $I_{n} \subset \pi_{n}[Q]$ hold for all but finitely many $n$. It follows that $I_{n} \neq \widetilde{E}_{n}$ for infinitely many $n$; hence, $I_{n}=E_{n}$ for infinitely many $n$. It follows that $E_{n} \subset \pi_{n}[Q]$ for infinitely many integers $n$. Let $k$ be a given integer not less than $N$. There is an integer $m$ not less than $k$ with the property that $E_{m} \subset \pi_{m}[Q]$. Then $E_{k} \subset f_{k}^{m}\left[E_{m}\right] \subset f_{k}^{m}\left[\pi_{m}[Q]\right]=\pi_{k}[Q]$. Consequently, $\pi_{k}[Q]$ intersects $\left\{a_{k}, b_{k}\right\}$ for $k$ not less than $N$. Therefore, $N$ is an E-number.

4.3. Points of irreducibility. For certain cases, points of irreducibility for $M$ can be found using the techniques developed in the previous sections. The remaining cases are covered by the following theorem. Its conclusion is that $M$ is irreducible about some two of four points. The proof gives a method for determining which two of the four are points of irreducibility for $M$.

THEOREM 19. If $N$ is an E-number for $\overline{z w}$ such that $f_{n}$ is not a two-pass map for integers $n$ not less than $N$, and $x$ and $y$ are points of $M$ such that $x_{N}=a_{N}, y_{N}=b_{N}$, and $\left\langle x_{n+1}, y_{n+1}\right\rangle$ is a type 4 pair with respect to $f_{n}$ for $n$ not less than $N$, then $M$ is irreducible about some two of $z, w, x$, and $y$.

Proof. Suppose $M$ is irreducible about the set $\{z, w, x, y\}$. Let $\alpha$ and $\beta$ be two points of $\{z, w, x, y\}$ such that there are infinitely many integers $n$ for which $\alpha_{n}=\min \left\{z_{n}, w_{n}, x_{n}, y_{n}\right\}$ and $\beta_{n}=\max \left\{z_{n}, w_{n}, x_{n}, y_{n}\right\}$. Then $\pi_{n}[\overline{\alpha \beta}]$ contains each of $z_{n}, w_{n}, x_{n}$, and $y_{n}$ for infinitely many positive integers $n$. It 
$\overline{\alpha \beta}$ contains each of $z, w, x, y$ and, therefore, $M$. So, it suffices to show that $M$ is irreducible about the set $\{z, w, x, y\}$.

Denote the subcontinuum of $M$ that is irreducible about $\{z, w, x, y\}$ by $M^{*}$. By Theorem 1, no proper subcontinuum of $M$ contains $\overline{z w}$ in its interior. Hence, by Theorem 12, every point of the complement of $\overline{z w}$ belongs to an indecomposable absolutely terminal subcontinuum of $M$ with interior. Since $\overline{z w} \subset M^{*}$, the conclusion of the theorem follows if $M^{*}$ contains every indecomposable absolutely terminal subcontinuum of $M$ with interior that is not contained in $\overline{z w}$. Suppose $Q$ is an indecomposable absolutely terminal subcontinuum of $M$ with interior that is not contained in $\overline{z w}$. Denote by $K$ the smallest of all integers $n$ not less than $N$ such that $\pi_{n}[Q]$ is nondegenerate.

It will be shown by induction that $\pi_{n}[Q] \cap\left\{x_{n}, y_{n}\right\} \cap\left\{a_{n}, b_{n}\right\}$ is nonempty for $N \leq n \leq K$. If $n=N$, the result is trivial since $\pi_{N}[Q]$ intersects $\left\{a_{N}, b_{N}\right\}$, and $\left\{a_{N}, b_{N}\right\}=\left\{x_{N}, y_{N}\right\}$. Suppose $\pi_{k-1}[Q] \cap\left\{x_{k-1}, y_{k-1}\right\} \cap\left\{a_{k-1}, b_{k-1}\right\}$ is nonempty for some integer $k$ satisfying $N<k \leq K$. Note that $\pi_{k}[Q]$ intersects $\left\{a_{k}, b_{k}\right\}$ since $N$ is an E-number. It will be assumed that $a_{k} \in \pi_{k}[Q]$ and $\pi_{k-1}[Q]=\left\{x_{k-1}\right\}$; the inductive step is similar if $b_{k} \in \pi_{k}[Q], \pi_{k-1}[Q]=$ $\left\{y_{k-1}\right\}$, or both. Then $f_{k-1}\left(a_{k}\right)=x_{k-1}$. Since $x_{k-1}$ is in $\left\{a_{k-1}, b_{k-1}\right\}$, and $f_{k-1}\left[a_{k}, \min \left\{x_{k}, y_{k}\right\}\right)$ does not intersect both $\left\{x_{k-1}\right\}$ and $\left\{a_{k-1}, b_{k-1}\right\}$, it follows that either $x_{k}=a_{k}$ or $y_{k}=a_{k}$. The coordinates $x_{N}$ and $y_{N}$ are not equal, so $x_{k-1}$ and $y_{k-1}$ are also not equal. Then $x_{k}=a_{k}$ since $f_{k-1}\left(a_{k}\right)=x_{k-1}$. Consequently, $\pi_{k}[Q] \cap\left\{x_{k}, y_{k}\right\} \cap\left\{a_{k}, b_{k}\right\}$ is nonempty.

Since $\pi_{K}[Q]$ is nondegenerate and $\pi_{K}[Q] \cap\left\{x_{K}, y_{K}\right\}$ is nonempty, it follows that $\operatorname{int}\left(\pi_{K}[Q]\right)$ contains one of $x_{K}$ and $y_{K}$. Then by Theorem 16 , $\operatorname{int}\left(\pi_{n}[Q]\right)$ intersects $\left\{x_{n}, y_{n}\right\}$ for $n$ not less than $K$. Since $\left\langle x_{n+1}, y_{n+1}\right\rangle$ is a type 4 pair with respect to $f_{n}$ for $n$ not less than $K$, it is a pair of one of type 1 , type 2 , or type 3 with respect to $f_{n}$ as well. Therefore, by Theorem 14 , $\overline{x y}$ contains $Q$. Since $\overline{x y} \subset M^{*}$, it follows that $M^{*}$ contains $Q$.

Summary. What follows is an outline of the procedure developed in this paper for obtaining points of irreducibility for $M$ in all cases.

1. If the inverse sequence $\left\{\left[a_{n}, b_{n}\right], f_{n}\right\}$ has infinitely many two-pass maps, then $M$ is indecomposable; hence, $M$ is irreducible between any pair of points $x$ and $y$ for which $\left\langle x_{n}, y_{n}\right\rangle$ is a pair of type 1 , type 2 , or type 3 for each positive integer $n$ by Corollary 14.1.

2. If $\left\{\left[a_{n}, b_{n}\right], f_{n}\right\}$ has zero or finitely many two-pass maps, and $M$ contains no imagewise nondecreasing E-sequence, then $z$ and $w$ are points of irreducibility for $M$ by Theorem 17 .

3 . If $\left\{\left[a_{n}, b_{n}\right], f_{n}\right\}$ has zero or finitely many two-pass maps, and $M$ contains an imagewise nondecreasing E-sequence, then Theorem 18 may be used to obtain an E-number for $\overline{z w}$, and Theorem 19 may be used to find points of irreducibility for $M$. 


\section{References}

[1] D. E. Bennett and J. B. Fugate, Continua and their non-separating subcontinua, Dissertationes Math. 149 (1977).

[2] R. H. Bing, Snake-like continua, Duke Math. J. 18 (1951), 653-663.

[3] W. D. Collins, A property of atriodic continua, Illinois J. Math. 28 (1984), 480-486.

[4] J. R. Isbell, Embeddings in inverse limits, Ann. of Math. 70 (1959), 73-84.

[5] D. P. Kuykendall, Irreducibility in inverse limits of intervals, Master's Thesis, Univ. of Houston, 1969.

[6] - Irreducibility and indecomposability in inverse limits, Fund. Math. 80 (1973), 265-270.

[7] M. A. Owens, Extremal continua: a class of non-separating subcontinua, Topology Appl. 23 (1986), 263-270.

[8] R. H. Sorgenfrey, Concerning triodic continua, Amer. J. Math. 66 (1944), $439-460$.

Department of Mathematics and Statistics

University of Missouri-Rolla

Rolla, MO 65409-0020, U.S.A.

E-mail: dryden@umr.edu

Received 15 August 1998;

in revised form 25 April 2000 